\title{
Nonmonotonic reasoning with normative conflicts in multi-agent deontic logic
}

\author{
Mathieu Beirlaen and Christian Straßer \\ Centre for Logic and Philosophy of Science \\ Ghent University \\ Blandijnberg 2, 9000 Ghent, Belgium \\ Phone: ++ 3292643979 \\ \{mathieu.beirlaen, christian.strasser\}@ugent.be
}

November 13, 2012

\section{Introduction}

We present proof-theoretical and semantical characterizations of two multi-agent deontic logics for dealing with normative conflicts. The resulting logics $\mathbf{P} \mathbf{M D L} \mathbf{L}^{\mathbf{r}}$ and $\mathbf{P} \mathbf{M D L} \mathbf{m}^{\mathbf{m}}$ are non-standard in at least two respects. First, they are nonclassical in the sense that they invalidate some inferences of the propositional fragment of Classical Logic (CL). Consequently, they also invalidate certain inferences of so-called Standard Deontic Logic (cfr. infra). The upshot of this non-classicality is that these logics consistently accommodate normative conflicts. Second, $\mathbf{P} \mathbf{M D L} \mathbf{r}^{\mathbf{r}}$ and $\mathbf{P} \mathbf{M D L} \mathbf{m}^{\mathbf{m}}$ are non-monotonic: previously derived conclusions may be withdrawn in the light of new premises. As such, these systems closely mirror actual normative and agentive reasoning.

Next to the usual connectives $\neg, \vee, \wedge, \supset$, and $\equiv$, we make use of a set of modal operators for bringing about collective actions, and of two deontic operators for mandatory and permitted states of affairs. We work within a simplified a-temporal framework from which we exclude e.g. authorities and utilities of obligations, knowledge and beliefs of agents and groups, etc.

The presentation of $\mathbf{P} \mathbf{M D L} \mathbf{L}^{\mathbf{r}}$ and $\mathbf{P} \mathbf{M D L} \mathbf{m}^{\mathbf{m}}$ proceeds in various steps. First, we define the monotonic, supraclassical multi-agent logic of action ML (Section 2 ). We illustrate how this logic deals with collective actions and discuss some further properties of our agentive modal operators. In Section 3 we extend ML with deontic modalities. The resulting logic is called MDL. We discuss some interesting properties of MDL related to collective obligations and (chains of) commands.

Next, we weaken MDL in order to consistently model less idealized settings in which intra- and interpersonal normative conflicts occur. In Section 4 we define the logic PMDL, a paraconsistent (yet monotonic) weakening of 
MDL that avoids explosion when faced with agents and groups with conflicting directives.

Although PMDL consistently deals with normative conflicts, it is too weak to account for many intuitive everyday normative inferences. That is why we non-monotonically strengthen PMDL in Section 5. This strengthening takes place within the adaptive logics framework for non-monotonic reasoning. The strengthening results in the adaptive logics $\mathbf{P} \mathbf{M D L} \mathbf{r}^{\mathbf{r}}$ and $\mathbf{P M D L}{ }^{\mathbf{m}}$, two logics that are intermediate in inferential power between the systems PMDL and MDL. $\mathbf{P M D L}^{\mathbf{r}}$ and $\mathbf{P M D L}{ }^{\mathbf{m}}$ approximate the classical setting in the sense that they take normative conflicts to be false whenever the premises allow for it. In doing so, $\mathbf{P} \mathbf{M D L} \mathbf{m}^{\mathbf{m}}$ is slightly more powerful than $\mathbf{P} \mathbf{M D L} \mathbf{L}^{\mathbf{r}}$.

This paper fits within the larger project of adaptive deontic logics devised for consistently accommodating normative conflicts (see e.g. [7, 29, 39, 41]). It improves on earlier work presented in [6]. We compare the logics $\mathbf{P} \mathbf{M D L} \mathbf{L}^{\mathbf{r}}$ and $\mathbf{P M D L}^{\mathbf{m}}$ to both logics of action and adaptive deontic logics in Section 6.

\section{ML, a simple multi-agent logic of action}

\subsection{Definition}

\subsubsection{Language and conventions}

We use a denumerable set $\mathcal{P}$ of propositional constants (atoms) $p, q, r, \ldots$, and a finite non-empty set $I=\left\{i_{1}, \ldots, i_{n}\right\}$ of agents. Since we will in the remainder often refer to groups of agents $J$ in $I$, i.e. non-empty subsets of $I$, the following notation is useful for this: $J \subseteq_{\varnothing} I$ iff $J \neq \varnothing$ and $J \subseteq I$. We also introduce the notation $J \subset_{\varnothing} I$ for denoting proper non-empty subsets $J$ of $I: J \subset_{\varnothing} I$ iff $J \neq \varnothing$ and $J \subset I$. Where $J \subseteq_{\varnothing} I$, the language $\mathcal{L}_{\mathbf{M L}}$ of $\mathbf{M L}$ is defined recursively as follows:

$$
\begin{aligned}
\mathcal{L}_{\mathrm{ML}}:= & \langle\mathcal{P}\rangle|\perp| \neg\left\langle\mathcal{L}_{\mathrm{ML}}\right\rangle\left|\left\langle\mathcal{L}_{\mathrm{ML}}\right\rangle \vee\left\langle\mathcal{L}_{\mathrm{ML}}\right\rangle\right|\left\langle\mathcal{L}_{\mathrm{ML}}\right\rangle \wedge\left\langle\mathcal{L}_{\mathrm{ML}}\right\rangle\left|\square_{J}\left\langle\mathcal{L}_{\mathrm{ML}}\right\rangle\right| \\
& \diamond_{J}\left\langle\mathcal{L}_{\mathrm{ML}}\right\rangle
\end{aligned}
$$

Note that we do not define the $\diamond_{J^{-}}$-operators in terms of their dual $\square_{J^{-}}$ operators. Instead, the diamond operators are primitive in our language. The reason for this will become clear in Section 4 . Where $A, B \in \mathcal{L}_{\mathrm{ML}}$, we define the implication by $A \supset B=\mathrm{df}_{\mathrm{df}} \neg A \vee B$ and the equivalence relation by $A \equiv B=\mathrm{df}_{\mathrm{df}}$ $(A \supset B) \wedge(B \supset A)$. A formula $\square_{J} A$ is interpreted as "group or agent $J$ brings about $A$ by a joint effort". A formula $\diamond_{J} A$ is interpreted (rather weakly) as " $A$ is consistent with the (joint) actions of group or agent $J$ " (cfr. Section 2.2).

Where $i \in J$, we abbreviate $\square_{\{i\}}$ as $\square_{i}$, and $\diamond_{\{i\}}$ as $\diamond_{i}$. Unless stated differently, we presuppose throughout this section that $A, B \in \mathcal{L}_{\mathrm{ML}}, \Gamma \subseteq \mathcal{L}_{\mathrm{ML}}$, and $J, K \subseteq_{\varnothing} I$. 


\subsubsection{Axiomatization}

ML is axiomatized by adding the following axiom schemata and rules to the propositional fragment of classical logic $(\mathbf{C L})$ :

$$
\begin{array}{r}
\square_{J}(A \supset B) \supset\left(\square_{J} A \supset \square_{J} B\right) \\
\square_{J} A \supset \square_{J} \square_{J} A \\
\square_{J} A \supset A \\
\diamond_{J} A \equiv \neg \square_{J} \neg A \\
\text { If } \vdash A, \text { then } \vdash \square_{J} A
\end{array}
$$

We write $\Gamma \vdash_{\mathrm{ML}} A$ iff there are $B_{1}, \ldots, B_{n}$ where $B_{n}=A$ and for each $i<n$, $B_{i} \in \Gamma$ or $B_{i}$ is an instance of an axiom of $\mathbf{M L}$, or $B_{i}$ is the result of applying a rule of $\mathbf{M L}$ to some $B_{j_{1}}, \ldots, B_{j_{m}}$ where $j_{1}, \ldots, j_{m}<i$.

The modal operators of $\mathbf{M L}$ are $\mathbf{S 4}$-operators. In agreement with the characterization of the $\diamond_{J}$-operators as separate modal operators not defined in terms of their duals (cfr. supra), we also need $\left(\mathrm{ADf} \diamond_{J}\right)$ in order to obtain the usual properties for the diamond operators.

\subsubsection{Semantics}

An ML-model is a tuple $\left\langle W,\left\langle R_{J}\right\rangle_{J \subseteq_{\varnothing I}, v}, @\right\rangle$, where $W$ is a set of points referred to as 'worlds', where each $R_{J} \subseteq W \times W$ is a transitive and reflexive accessibility relation ${ }^{1}$, where $v: \mathcal{P} \rightarrow \wp(W)$ is an assignment function, and where @ $\in W$ is the 'home' or 'actual' world.

Truth at a world $w$ is defined as follows:

$(\mathrm{CP}) \quad$ where $A \in \mathcal{P}, M, w \vDash A$ iff $A \in v(w)$

(C^) $\quad M, w \vDash A \wedge B$ iff $M, w \vDash A$ and $M, w \vDash B$

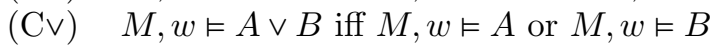

$\left(\mathrm{C}_{\neg}\right) \quad M, w \vDash \neg A$ iff $M, w \neq A$

$(\mathrm{C} \perp) \quad M, w \neq \perp$

$\left(\mathrm{C} \square_{J}\right) \quad M, w \vDash \square_{J} A$ iff for all $w^{\prime}$ such that $R_{J} w w^{\prime}, M, w^{\prime} \vDash A$

$\left(\mathrm{C} \diamond_{J}\right) \quad M, w \vDash \diamond_{J} A$ iff there is a $w^{\prime}$ such that $R_{J} w w^{\prime}$ and $M, w^{\prime} \vDash A$

An ML-model $M$ verifies $A\left(M \vDash_{\mathbf{M L}} A\right)$ iff $M$, @ $\vDash A$. $M$ is an ML-model of $\Gamma$ iff $M$ is an ML-model and $M \vDash_{\text {ML }} A$ for all $A \in \Gamma$. Moreover, $\vdash_{\text {ML }} A$ iff all ML-models verify $A$, and $\Gamma \Vdash_{\text {ML }} A$ iff all ML-models of $\Gamma$ verify $A$.

\subsection{Further discussion}

As mentioned above, we read $\square_{J} A$ as "group or agent $J$ brings about $A$ by a joint effort". $\diamond_{J} A$ is read as " $A$ is consistent with $J$ 's actions", instead of

\footnotetext{
${ }^{1} R$ is transitive iff, for each $w, w^{\prime}$, and $w^{\prime \prime}$, whenever $R w w^{\prime}$ and $R w^{\prime} w^{\prime \prime}$, also $R w w^{\prime \prime} ; R$ is reflexive iff, for each $w, R w w$.
} 
the stronger " $J$ has the ability to bring about $A$ ". ${ }^{2}$ The reason for this weaker reading has to do with the following inferences:

$$
\begin{gathered}
\diamond_{J}(A \vee B) \vdash_{\mathrm{ML}} \diamond_{J} A \vee \diamond_{J} B \\
A \vdash_{\mathrm{ML}} \diamond_{J} A
\end{gathered}
$$

Of (1) and (2), Kenny noted in [25] that these are too strong for formalizing the 'can' of ability. (1) is violated by anyone who has the ability to pick a card from a pack of cards without having the ability to pick a red card or the ability to pick a black one. (2) is violated by any hopeless darts player who by accident - hits the bull's eye but lacks the ability to repeat his deed [25, 36]. For this reason, we prefer our weaker reading of the $\diamond_{J}$-operators. We refer to [22, Sec. 2.3] for a more detailed treatment of (individual) ability.

As the modal operators of $\mathbf{M L}$ are $\mathbf{S 4}$-modalities, we can aggregate over actions:

$$
\square_{J} A \wedge \square_{J} B \vdash_{\mathrm{ML}} \square_{J}(A \wedge B)
$$

The opposite direction of (3) also holds:

$$
\square_{J}(A \wedge B) \vdash_{\mathrm{ML}} \square_{J} A \wedge \square_{J} B
$$

ML invalidates the stronger axiom schemata $\left(\mathrm{A} 5 \square_{J}\right)$ and $\left(\mathrm{AB} \square_{J}\right)$ :

$$
\begin{array}{r}
\diamond_{J} A \supset \square_{J} \diamond_{J} A \\
A \supset \square_{J} \diamond_{J} A
\end{array}
$$

$A$ 's being consistent with $J$ 's actions need not imply that $J$ brings it about that $A$ is consistent with his/her/its actions. Moreover, $A$ 's being the case need not imply that - for all agents and groups $J-J$ takes care (or brings it about) that $A$ is consistent with $J$ 's actions.

As indicated in Section 2.1.1, group actions are joint actions in ML. A formula $\square_{J} A$ is true only if all members of $J$ bring about $A$ together. In the ML-semantics, the actions of individuals and groups are represented by a set of accessibility relations all of which are structurally independent of one another. As a result, where $J \subseteq_{\varnothing} K$ :

$$
\begin{aligned}
& \square_{J} A \nvdash_{\mathrm{ML}} \square_{K} A \\
& \diamond_{J} A \nvdash_{\mathrm{ML}} \diamond_{K} A \\
& \square_{K} A \nvdash_{\mathrm{ML}} \square_{J} A \\
& \diamond_{K} A \nvdash_{\mathrm{ML}} \diamond_{J} A
\end{aligned}
$$

(5) and (6) illustrate that ML's agency operators do not allow for the inclusion of 'free riders' in their actions: for each action $\square_{J} A$, each member of the group $J$ is essential to $J$ 's bringing about $A .^{3}$

\footnotetext{
${ }^{2}$ Note that due to $\left(\mathrm{NEC}_{J}\right)$ we have $\square_{J} A$ for all ML-theorems $A$, for every $J \subseteq_{\varnothing} I$. This adds a non-deliberative flavor to our $\square_{J}$ operator similar to the non-deliberative character of the Chellas-stit (see e.g., [22]). In view of this a more refined reading of $\square_{J} A$ is "group or agent $J$ brings about $A$ (by a joint effort) or $A$ is logically necessary".

${ }^{3}$ The concept of free riders is borrowed from [8].
} 
(7) and (8) illustrate that in ML a group's actions are not carried over to its members, or to smaller subgroups. If members of a group were to inherit the actions of the groups to which they belong, then if they would realize something as a team, it would follow that each of them realizes it separately. This is clearly unwanted, since, for instance, none of the agents might to be capable of realizing the action by herself.

The only constraints present on the actions of individuals and groups in ML is that they need to be consistent with the actions of other agents and groups, and with the facts. For all $J, K$ :

$$
\begin{gathered}
A \vdash \mathrm{ML} \\
A \vdash_{J} A \\
\left.\square_{J} A \vdash_{\mathrm{ML}}\right\urcorner_{J} \neg A \\
\square_{J} A \vdash \mathrm{ML} \\
\neg \square_{K} \neg A
\end{gathered}
$$

Following [22, Sec. 2.3.3], we define an agent or group's refraining from $A$ as $\square_{J} \neg \square_{J} A$. Refrainment is stronger than simple non-action:

$$
\square_{J} \neg \square_{J} A \vdash_{\mathrm{ML}} \neg \square_{J} A
$$

(13) follows immediately by $\left(\operatorname{AT} \square_{J}\right)$. Its converse, however, does not hold in ML:

$$
\neg \square_{J} A \nvdash_{\mathrm{ML}} \square_{J} \neg \square_{J} A
$$

This is as it should be: in not bringing about a state of affairs, we need not 'actively' do so. Von Wright notes that this is especially true in situations in which acting so-and-so is beyond our capacity. For example, while it may be true that an agent does not alter the course of a tornado, it seems incorrect to say that she refrains from doing so [42].

The $\square_{J}$-operator is not a 'deliberative' action operator in the sense of [24], since for instance the following not so intuitive formulas are ML-theorems:

$$
\begin{aligned}
& \vdash_{\mathrm{ML}} \square_{J}(A \vee \neg A) \\
& \vdash_{\mathrm{ML}} \square_{J}\left(\square_{J} A \vee \neg \square_{J} A\right)
\end{aligned}
$$

If we were to add to ML a modal operator “ $\square$ " for representing (physical) necessity and call the resulting logic $\mathbf{M} \mathbf{L}^{\prime}$, then, in line with the literature on deliberative agency, a deliberative agency-operator $\triangle_{J}$ can be defined in $\mathbf{M L}^{\prime}$ by $\triangle_{J} A==_{\mathrm{df}}\left(\square_{J} A \wedge \neg \square A\right)$. The analogues to (15) and (16) are invalid for this new operator:

$$
\begin{aligned}
& { }^{\mathrm{ML}^{\prime}} \triangle_{J}(A \vee \neg A) \\
& { }_{\mathrm{ML}^{\prime}} \triangle_{J}\left(\triangle_{J} A \vee \neg \triangle_{J} A\right)
\end{aligned}
$$

For convenience, we will in the remainder continue to use the $\square_{J}$-operators instead of the more involving $\Delta_{J}$-operators. 


\section{Adding deontic modalities: the logic MDL}

\subsection{Definition}

\subsubsection{Language}

The language $\mathcal{L}_{\text {MDL }}$ of MDL is obtained by adding the deontic operators $O$ and $\mathrm{P}$ to the language of $\mathrm{ML}$ :

$$
\begin{aligned}
\mathcal{L}_{\mathrm{MDL}}:= & \left\langle\mathcal{L}_{\mathrm{ML}}\right\rangle\left|\neg\left\langle\mathcal{L}_{\mathrm{MDL}}\right\rangle\right|\left\langle\mathcal{L}_{\mathrm{MDL}}\right\rangle \vee\left\langle\mathcal{L}_{\mathrm{MDL}}\right\rangle\left|\left\langle\mathcal{L}_{\mathrm{MDL}}\right\rangle \wedge\left\langle\mathcal{L}_{\mathrm{MDL}}\right\rangle\right| \\
& \square_{J}\left\langle\mathcal{L}_{\mathrm{MDL}}\right\rangle\left|\diamond_{J}\left\langle\mathcal{L}_{\mathrm{MDL}}\right\rangle\right| \mathrm{O}\left\langle\mathcal{L}_{\mathrm{MDL}}\right\rangle \mid \mathrm{P}\left\langle\mathcal{L}_{\mathrm{MDL}}\right\rangle
\end{aligned}
$$

As for ML, we do not define the P-operator as the dual of the O-operator, but add it separately to the language of MDL. The reason for doing so will become clear in Section 4.

Note that, unlike e.g. Horty's treatment in [22], only one obligation operator is introduced in MDL. Obligations are made agent-relative by suffixing a deontic operator with an agentive modality.

Where $A \in \mathcal{L}_{\text {MDL }}$, a formula $\mathrm{O} A$ is read as "it is obligatory that $A$ ". $\mathrm{P} A$ is read as "it is permitted that $A$ ".

Unless stated differently, we presuppose throughout this section that $A, B \in$ $\mathcal{L}_{\mathrm{MDL}}, \Gamma \subseteq \mathcal{L}_{\mathrm{MDL}}$, and $J, K \subseteq_{\varnothing} I$.

\subsubsection{Axiomatization}

MDL is axiomatized by adding to ML the axiom schemata (AKO), (ADO), (ADfP), and the rule (NECO):

$$
\begin{array}{r}
\mathrm{O}(A \supset B) \supset(\mathrm{O} A \supset \mathrm{O} B) \\
\mathrm{O} A \supset \mathrm{P} A \\
\mathrm{P} A \equiv \neg \mathrm{O} \neg A \\
\text { If } \vdash A, \text { then } \vdash \mathrm{O} A
\end{array}
$$

We write $\Gamma \vdash_{\text {MDL }} A$ iff there are $B_{1}, \ldots, B_{n}$ where $B_{n}=A$ and for each $i<n$, $B_{i} \in \Gamma$ or $B_{i}$ is an instance of an axiom of $\mathbf{M D L}$, or $B_{i}$ is the result of applying a rule of MDL to some $B_{j_{1}}, \ldots, B_{j_{m}}$ where $j_{1}, \ldots, j_{m}<i$.

(AKO), (ADO), (ADfP), and (NECO) give us full 'Standard Deontic Logic' (SDL) for the deontic operators. ${ }^{4}$

\subsubsection{Semantics}

An MDL-model is a tuple $\left\langle W,\left\langle R_{J}\right\rangle_{J \subseteq_{\varnothing} I}, R_{\mathrm{O}}, v, @\right\rangle$, where $W,\left\langle R_{J}\right\rangle_{J \subseteq_{\varnothing} I}, v$ and @ are as before, and where $R_{\mathrm{O}} \subseteq W \times W$ is a serial accessibility relation. ${ }^{5}$ Truth at a world $w$ is defined by adding to clauses $(\mathrm{CP})-\left(\mathrm{C} \diamond_{J}\right)$ from Section 2.1.3 the clauses $(\mathrm{CO})$ and $(\mathrm{CP})$ :

\footnotetext{
${ }^{4} \mathbf{S D L}$ is sometimes also called $\mathbf{D}$ or $\mathbf{K D}$ since it extends the basic normal modal logic $\mathbf{K}$ by the axiom schema (ADO), which is often simply called (D).

${ }^{5} R$ is serial iff for each world $w$ there is some $w^{\prime}$ such that $R w w^{\prime}$.
} 
(CO) $\quad M, w \vDash \mathrm{O} A$ iff for all $w^{\prime}$ such that $R_{\mathrm{O}} w w^{\prime}, M, w^{\prime} \vDash A$

(CP) $\quad M, w \vDash \mathrm{P} A$ iff for some $w^{\prime}$ such that $R_{\mathrm{O}} w w^{\prime}, M, w^{\prime} \vDash A$

An MDL-model $M$ verifies $A\left(M \vDash_{\text {MDL }} A\right)$ iff $M$, @ $\vDash A$. $M$ is an MDLmodel of $\Gamma$ iff $M$ is an MDL-model and $M \vDash_{\text {MDL }} A$ for all $A \in \Gamma$. Moreover, $\Vdash_{\text {MDL }} A$ iff all MDL-models verify $A$, and $\Gamma \Vdash$ MDL $A$ iff all MDL-models of $\Gamma$ verify $A$.

\subsection{Discussion}

As the O-operator too is a normal modal operator, we can aggregate over obligations:

$$
\begin{gathered}
\mathrm{O} A \wedge \mathrm{O} B \vdash_{\mathrm{MDL}} \mathrm{O}(A \wedge B) \\
\mathrm{O} \square_{J} A \wedge \mathrm{O} \square_{K} B \vdash_{\mathrm{MDL}} \mathrm{O}\left(\square_{J} A \wedge \square_{K} B\right) \\
\mathrm{O} \square_{J} A \wedge \mathrm{O} \square_{J} B \vdash_{\mathrm{MDL}} \mathrm{O} \square_{J}(A \wedge B)
\end{gathered}
$$

Where $J \subseteq_{\varnothing} K$, the deontic analogues of (5)-(8) remain invalid in MDL:

$$
\begin{aligned}
& \mathrm{O} \square_{J} A \nvdash_{\mathrm{ML}} \mathrm{O} \square_{K} A \\
& \mathrm{O} \diamond_{J} A \nvdash_{\mathrm{ML}} \mathrm{O} \diamond_{K} A \\
& \mathrm{O} \square_{K} A \nvdash_{\mathrm{ML}} \mathrm{O} \square_{J} A \\
& \mathrm{O} \diamond_{K} A{{ }_{\mathrm{ML}}}^{\mathrm{O}} \diamond_{J} A
\end{aligned}
$$

And similarly for permissions. Thus, obligations and permissions too are not closed under weakening or strengthening via the addition or subtraction of agents to the group. Collective obligations of the kind interpreted by MDL are called strict collective obligations by Dignum \& Royakkers [12]. A strict collective obligation to bring about $A$ is satisfied only if all agents in the collective bring about $A$ together. ${ }^{6}$

Next to strict collective obligations, Dignum \& Royakkers also define weak collective obligations. A weak collective obligation to bring about $A$ is satisfied as soon as any subset of the collective brings about $A$. Given the language $\mathcal{L}_{\mathrm{MDL}}$, we can define an operator $\mathrm{O}^{\mathrm{w}}$ for expressing weak collective obligations as follows:

$$
\mathrm{O}^{\mathrm{w}} \square_{J} A=_{\mathrm{df}} \mathrm{O}\left(\mathrm{V}_{K \subseteq_{\varnothing} J} \square_{K} A\right)
$$

The weak collective obligation operator $\mathrm{O}^{\mathrm{w}}$ captures the intended meaning that if it is obligatory for a group of agents to bring about a certain state of affairs, then this state of affairs ought to be brought about by some subset of the group.

\footnotetext{
${ }^{6}$ Strict collective obligations should be distinguished from so-called general obligations: obligations pertaining to each individual in a group. An obligation to drive on the righthand side of the road, for instance, is a general, but not a (strict) collective obligation: it holds of each individual in a group, but not of the group itself. See $[1,19]$ for discussions on general obligations.
} 
Where $K \subset_{\varnothing} J$, an obligation $\square_{J} A$ is MDL-compatible with $\square_{K} A$. Thus, even if group $J$ has the strict collective obligation to bring about $A$, it might still be the case that some proper subset $K$ of $J$ (also) ought to bring about $A$. In [8], Belnap \& Perloff introduce an operator for strict joint agency by defining what it is for an agent to be essential for a (collective) action. Translated to our framework, we say that - where $J \subseteq_{\varnothing} I$ - an agent $i \in J$ is essential for $\square_{J} A$ iff $\left(\square_{J} A\right.$ and there is no $K \subset_{\varnothing} J$ such that $i \notin K$ and $\square_{K} A$ ).

Where $J, K \subseteq_{\varnothing} I$, we can define an operator $\mathrm{O}^{\mathrm{e}}$ for exclusive strict obligations:

$\mathrm{O}^{\mathrm{e}} \square_{J} A=_{\mathrm{df}} \mathrm{O} \square_{J} A \wedge\left(\wedge_{K \subset_{\varnothing} J} \neg \mathrm{O} \square_{K} A\right)$

Alternatively, we could say that a group $J$ has the exclusive strict obligation to bring about $A$ if all agents in $J$ are essential for $A$.

Where $J \subset_{\varnothing} K$, the following weakening and strengthening properties hold for operators $\mathrm{O}^{\mathrm{w}}$ and $\mathrm{O}^{\mathrm{e}}$ in MDL:

$$
\begin{gathered}
\mathrm{O}^{\mathrm{w}} \square_{J} A \vdash_{\mathrm{MDL}} \mathrm{O}^{\mathrm{w}} \square_{K} A \\
\mathrm{O}^{\mathrm{w}} \square_{K} A \nvdash_{\mathrm{MDL}} \mathrm{O}^{\mathrm{w}} \square_{J} A \\
\mathrm{O}^{\mathrm{e}} \square_{J} A \nvdash_{\mathrm{MDL}} \mathrm{O}^{\mathrm{e}} \square_{K} A \\
\mathrm{O}^{\mathrm{e}} \square_{K} A \nvdash_{\mathrm{MDL}} \mathrm{O}^{\mathrm{e}} \square_{J} A
\end{gathered}
$$

Another form of interaction between agents occurs when actions get nested or iterated. In line with the (literal) reading of $\square_{J} A$ and $\mathrm{O} A$, we read a formula $\square_{J} \mathrm{O} \square_{K} A$ as " $J$ brings it about that it is obligatory that $K$ brings it about that $A$ ". Alternatively, we can interpret this formula as " $J$ issues the obligation for $K$ to bring about $A "$.

$$
\mathrm{O} \square_{J} \mathrm{O} \square_{K} A \nvdash_{\mathrm{MDL}} \mathrm{O} \square_{J} A
$$

(30) expresses that if it is obligatory for $J$ to issue the obligation for $K$ to bring about $A$, then it need not be obligatory for $J$ to realize $A$. This is as it should be, since $J$ might realize his/her/their duty and issue the obligation to $K$, without $K$ realizing his/her/their duty to actually bring about $A$. Hence it is not up to $J$ to bring about $A$. Thus, we cannot derive $\mathrm{O} \square_{J} A$ from $\mathrm{O} \square_{J} \mathrm{O} \square_{K} A$.

So far, the treatment of actions, obligations, and action-obligation compounds by MDL seems fine. Things change, however, when we turn to more 'messy' settings in which the requirements on agents can conflict.

\section{Dealing with normative conflicts}

\subsection{MDL and normative conflicts}

In Sophocles' Antigone, Creon declares the burial of Antigone's brother Polyneices illegal on the grounds that he was a traitor to the city, and that his burial would mock the loyalists who defended the city. At the same time however, Antigone faces a religious and familial obligation to bury her brother [17, p. 4]. 
The conflicting obligations of Antigone and Creon to bury and not to bury Polyneices can be formalized as $\mathrm{O} \square_{a} B$ and $\mathrm{O}_{c_{c}} \neg B$ respectively (where ' $a$ ' abbreviates 'Antigone', ' $c$ ' abbreviates Creon, and ' $B$ ' abbreviates the statement "Polyneices is buried"). Conflicts between obligations for different agents or groups to bring about some state of affairs are called interpersonal conflicts in $[28,38]$.

Interpersonal obligation-obligation conflicts or OO-conflicts of the kind displayed above cannot be consistently formalized in MDL, due to the validity of (31). Where $J \neq K$ :

$$
\mathrm{O} \square_{J} A \wedge \mathrm{O} \square_{K} \neg A \vdash_{\text {MDL } \perp}
$$

Similarly for interpersonal obligation-permission conflicts or OP-conflicts:

$$
\mathrm{O} \square_{J} A \wedge \mathrm{P} \square_{K} \neg A \vdash_{\mathrm{MDL}} \perp
$$

As has been argued extensively by moral philosophers and deontic logicians, single agents and groups too can face (intra-personal) OO- or OP-conflicts (see e.g. $[13,16,27,43]$ ). An adult muslim living in Western Europe might for instance be permitted to drink alcohol (by law) and forbidden to drink alcohol (by his or her religion). However, such situations too cause explosion when formalized in MDL, due to the validity of:

$$
\begin{aligned}
& \mathrm{O} \square_{J} A \wedge \mathrm{O} \square_{J} \neg A \vdash_{\mathrm{MDL}} \perp \\
& \mathrm{O} \square_{J} A \wedge \mathrm{P} \square_{J} \neg A \vdash_{\mathrm{MDL}} \perp
\end{aligned}
$$

The same story applies to the slightly weaker inferences (35) and (36), and to 'nested' OO- or OP-conflicts:

$$
\begin{aligned}
& \mathrm{O} \square_{J} A \wedge \mathrm{O} \neg \square_{J} A \vdash \mathrm{MDL} \perp \\
& \mathrm{O} \square_{J} A \wedge \mathrm{P} \neg \square_{J} A \vdash \mathrm{MDL} \perp \\
& \mathrm{O} \square_{J} \mathrm{O} \square_{K} A \wedge \mathrm{O} \square_{J} \mathrm{O} \square_{K} \neg A \vdash_{\mathrm{MDL}} \perp \\
& \mathrm{O} \square_{J} \mathrm{O} \square_{K} A \wedge \mathrm{O} \square_{J} \mathrm{P} \square_{K} \neg A \vdash \mathrm{MDL} \perp
\end{aligned}
$$

In general, the following explosion principles are MDL-valid:

$$
\begin{array}{r}
\mathrm{O} A \wedge \mathrm{O} \neg A \vdash \mathrm{MDL} \perp \\
\mathrm{O} A \wedge \mathrm{P} \neg A \vdash \mathrm{MDL} \perp \\
\text { If } \vdash_{\mathrm{MDL}} \neg\left(A_{1} \wedge \ldots \wedge A_{n}\right), \text { then } \mathrm{O} \square_{J_{1}} A_{1} \wedge \ldots \wedge \mathrm{O} \square_{J_{n}} A_{n} \vdash \mathrm{MDL} \perp \\
\text { If } \vdash_{\mathrm{MDL}} \neg\left(A_{1} \wedge \ldots \wedge A_{n}\right), \text { then } \mathrm{O} \square_{J_{1}} A_{1} \wedge \ldots \wedge \mathrm{P} \square_{J_{n}} A_{n} \vdash \mathrm{MDL} \perp
\end{array}
$$

Unfortunately, real life is abundant with (inter- and intra-personal) OO- and OP-conflicts between (groups of) agents [26]. Hence we should be able to consistently accommodate such conflicts within our logic. In Section 4.2, we weaken MDL to a logic that invalidates the explosion principles (39)-(42). 


\subsection{Enters paraconsistency: the logic PMDL}

The solution adopted here for the problem of consistently accommodating normative conflicts, is to weaken the negation connective of MDL to a paraconsistent negation connective. A negation connective " $\neg$ " is paraconsistent if it invalidates the Ex Contradictione Quodlibet (ECQ) principle:

$$
A, \neg A \vdash B
$$

Below we introduce a logic that weakens " $\neg$ " to a paraconsistent negation connective, namely the logic PMDL. PMDL no longer strengthens CL. Rather, it is built on top of the propositional fragment of the much weaker paraconsistent $\operatorname{logic} \mathbf{L P}{ }^{7}$

For reasons of transparency, we first characterize PMDL semantically. The PMDL-semantics differs from that of MDL in that (i) we broaden the range of the assignment function $v$ so that it includes the set of literals $\mathcal{P}\urcorner=\{A \cup \neg A \mid$ $A \in \mathcal{P}\}$, i.e. we define $v: \mathcal{P}\urcorner \rightarrow \wp(W)$, (ii) we replace clause $\left(\mathrm{C}_{\neg}\right)$ by $\left(\mathrm{C}_{\neg}^{\prime}\right)$ and add de Morgan's laws to the semantics (clauses $\left(\mathrm{C}_{\neg \neg)}-(\mathrm{C}-\neg \vee)\right.$ ), and (iii) we add clauses $\left(\mathrm{C} \neg \square_{J}\right),\left(\mathrm{C} \neg \diamond_{J}\right),(\mathrm{C} \neg \mathrm{O})$, and $(\mathrm{C} \neg \mathrm{P})$ which give us the usual interrelations between dual operators. Thus, we keep clauses $(\mathrm{CP}),(\mathrm{C} \wedge),(\mathrm{C} \vee)$, $(\mathrm{C} \perp),\left(\mathrm{C} \square_{J}\right),(\mathrm{C} \diamond J),(\mathrm{CO})$, and $(\mathrm{CP})$ and add the following:

$$
\begin{aligned}
\left(\mathrm{C}^{\prime}\right) & \text { Where } A \in \mathcal{P}, M, w \vDash \neg A \text { iff } M, w \vDash A \text { or } \neg A \in v(w) \\
(\mathrm{C} \neg \neg) & M, w \vDash \neg \neg A \text { iff } M, w \vDash A \\
(\mathrm{C} \neg \wedge) & M, w \vDash \neg(A \wedge B) \text { iff } M, w \vDash \neg A \vee \neg B \\
(\mathrm{C} \neg \vee) & M, w \vDash \neg(A \vee B) \text { iff } M, w \vDash \neg A \wedge \neg B \\
\left(\mathrm{C}_{\neg} \square_{J}\right) & M, w \vDash \neg \square_{J} A \text { iff } M, w \vDash \diamond_{J} \neg A \\
\left(\mathrm{C} \neg \diamond_{J}\right) & M, w \vDash \neg \diamond_{J} A \text { iff } M, w \vDash \square \neg \neg A \\
(\mathrm{C} \neg \mathrm{O}) & M, w \vDash \neg \mathrm{O} A \text { iff } M, w \vDash \mathrm{P} \neg A \\
(\mathrm{C} \neg \mathrm{P}) & M, w \vDash \neg \mathrm{P} A \text { iff } M, w \vDash \mathrm{O} \neg A
\end{aligned}
$$

As before, a PMDL-model $M$ verifies $A\left(M \vDash_{\mathbf{P M D L}} A\right)$ iff $M$, $@ \vDash A . M$ is a PMDL-model of $\Gamma$ iff $M$ is a PMDL-model and $M \vDash_{\mathbf{P M D L}} A$ for all $A \in \Gamma$. Moreover, $\vdash_{\text {PMDL }} A$ iff all PMDL-models verify $A$, and $\Gamma \Vdash$ PMDL $A$ iff all PMDL-models of $\Gamma$ verify $A$.

The addition of clauses $\left(\mathrm{C} \neg \square_{J}\right),\left(\mathrm{C} \neg \diamond_{J}\right),(\mathrm{C} \neg \mathrm{O})$, and $(\mathrm{C} \neg \mathrm{P})$ is necessary in order to guarantee the interdefinability of these modal operators. If, for instance, the P-operator were simply defined as the dual of the O-operator (i.e. $\left.\mathrm{P} A={ }_{\mathrm{df}} \neg \mathrm{O} \neg A\right)$, then, due to the paraconsistency of " $\neg$ " we would no longer be able to derive $\mathrm{P} \neg A$ from $\neg \mathrm{O} A$. Similarly for the $\square_{J^{-}}$and $\diamond_{J}$-operators. This is why all modalities are primitive in our language, and why extra semantic clauses are added in order to guarantee their usual interrelations.

Syntactically, the negation connective of $\mathbf{L P}$ is defined by de Morgan's laws and Excluded Middle (EM):

$$
\vdash A \vee \neg A
$$

\footnotetext{
${ }^{7}$ LP was devised by Priest [32] and abbreviates 'Logic of Paradox'. See [34] for more information.
} 
Since LP no longer validates (ECQ), it can consistently allow for contradictions $A \wedge \neg A$. A consequence of this weakening is that LP invalidates Modus Ponens, due to the definition of the implication connective in terms of the negation and disjunction connectives, cfr. Section 2.1.1. A full syntactical characterization of LP is contained in Section A of the Appendix.

Where $\langle\varpi, \diamond\rangle \in\left\{\langle\mathrm{O}, \mathrm{P}\rangle,\left\langle\square_{J}, \diamond_{J}\right\rangle \mid J \subseteq_{\varnothing} I\right\}$, the logic PMDL is defined by adding to $\mathbf{L P}$ the rules $\left(4 \square_{J}\right)-\left(\mathrm{T} \diamond_{J}\right)$ for every $J \subseteq_{\varnothing} I$, (DO), and (ANDø)$(\mathrm{INH} \odot)$ :

$$
\begin{aligned}
& \square_{J} A \vdash \square_{J} \square_{J} A \quad\left(4 \square_{J}\right) \\
& \diamond_{J} \diamond_{J} A \vdash \diamond_{J} A \quad\left(4 \diamond_{J}\right) \\
& \square_{J} A \vdash A \quad\left(\mathrm{~T} \square_{J}\right) \\
& A \vdash \diamond_{J} A \quad\left(\mathrm{~T} \diamond_{J}\right) \\
& \mathrm{O} A \vdash \mathrm{P} A \quad(\mathrm{DO}) \\
& \varpi A, \varpi B \vdash \odot(A \wedge B) \quad(\mathrm{AND}) \\
& \bullet A, \diamond B \vdash \odot(A \wedge B) \quad\left(\mathrm{AND}^{\prime} \odot\right) \quad \text { If } A \vdash B \text {, then } \bullet A \vdash \bullet B \text {. } \\
& \text { If } A \vdash B \text {, then } \diamond A \vdash \diamond B \text {. }
\end{aligned}
$$

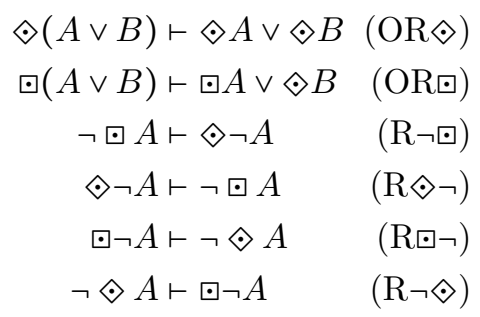

In the case of (INH๑) and (INH↔) we also allow for the case that $A$ is the empty string, in which case we stipulate that also $\bullet A$ resp. $\odot A$ is the empty string. We write $\Gamma \vdash$ PMDL $A$ iff there are $B_{1}, \ldots, B_{n}$ where $B_{n}=A$ and for each $i<n, B_{i} \in \Gamma$ or $B_{i}$ is an instance of an axiom of PMDL, or $B_{i}$ is the result of applying a rule of PMDL to some $B_{j_{1}}, \ldots, B_{j_{m}}$ where $j_{1}, \ldots, j_{m}<i$.

Note that all of the rules of PMDL are MDL-valid. As we will illustrate below, PMDL is strictly weaker than MDL. The reason why the properties of PMDL are introduced as rules - and not as axiom schemata - is that the implication connective of PMDL is not detachable: Modus Ponens is invalid in PMDL. For instance, if instead of $\left(\mathrm{T} \square_{J}\right)$ only its weaker variant $\square_{J} A \supset A$ were to hold, then $A$ would not be PMDL-derivable from $\square_{J} A$ and $\square_{J} A \supset A$.

Theorem 1. $\Gamma \vdash_{\text {PMDL }} A$ iff $\Gamma \vDash_{\text {PMDL }} A$.

A proof of Theorem 1 is contained in Section B of the Appendix.

In accordance with the goal set out for this logic, PMDL consistently tolerates all types of normative conflicts mentioned in Section 4.1; in other words, PMDL invalidates the explosion principles (39)-(42):

$$
\begin{array}{r}
\mathrm{O} A \wedge \mathrm{O} \neg A \nvdash_{\text {PMDL }} \perp \\
\mathrm{O} A \wedge \mathrm{P} \neg A r_{\text {PMDL }} \perp \\
\text { If } \vdash_{\mathrm{MDL}} \neg\left(A_{1} \wedge \ldots \wedge A_{n}\right), \text { then } \mathrm{O} \square_{J_{1}} A_{1} \wedge \ldots \wedge \mathrm{O} \square_{J_{n}} A_{n} \forall_{\text {PMDL }} \perp \\
\text { If } \vdash_{\mathrm{MDL}} \neg\left(A_{1} \wedge \ldots \wedge A_{n}\right), \text { then } \mathrm{O} \square_{J_{1}} A_{1} \wedge \ldots \wedge \mathrm{P} \square_{J_{n}} A_{n} \forall_{\text {PMDL }} \perp
\end{array}
$$

\subsection{A price to pay?}

Unfortunately, our story does not end here. Although PMDL provides a consistent treatment of normative conflicts, this treatment comes at a high price. 
Not only does PMDL invalidate inferences (43)-(46) (as was desired); alas it also invalidates many other - less unwanted - MDL-valid inferences, e.g.:

$$
\begin{gathered}
\mathrm{O} \square_{J} \neg A, \mathrm{O} \square_{J}(A \vee B){ }_{\text {PMDL }} \mathrm{O} \square_{J} B \\
\mathrm{O} \neg A, \mathrm{O}(A \vee B) \text { PMDL } \mathrm{O} B \\
\square_{J} A, \square_{J}(\neg A \vee B) \text { PMDL }_{{ }_{J}} B
\end{gathered}
$$

In general, the Disjunctive Syllogism and Modus Ponens rules fail in PMDL:

$$
\begin{gathered}
A, \neg A \vee B \nleftarrow \mathbf{P M D L} B \\
A, A \supset B \nleftarrow \mathbf{P M D L} B
\end{gathered}
$$

This is a very high price to pay for the conflict-tolerance of PMDL. PMDL is way too poor to account for our everyday normative and non-normative, agentive and non-agentive reasoning.

Thus PMDL suffers from a trade-off. In Section 5 we propose to overcome this trade-off by non-monotonically strengthening PMDL within the standard format for adaptive logics [3]. The resulting adaptive logics $\mathbf{P} \mathbf{M D L} \mathbf{r}^{\mathbf{r}}$ and $\mathbf{P M D L}^{\mathbf{m}}$ interpret a given premise set 'as consistently as possible'. On the one hand, these logics allow us to defeasibly apply all MDL-valid inference steps on the condition that the formulas to which we apply them behave consistently. On the other hand, $\mathbf{P} \mathbf{M D L} \mathbf{L}^{\mathbf{r}}$ and $\mathbf{P} \mathbf{M D L} \mathbf{L}^{\mathbf{m}}$ remain fully conflict-tolerant.

\section{Adaptive multi-agent deontic logic}

\subsection{Proof-theoretical characterization of the logics $\mathrm{PMDL}^{\mathrm{r}}$ and $\mathrm{PMDL}^{\mathrm{m}}$}

\subsubsection{Three generic rules of inference}

An adaptive logic is characterized by three elements: a so-called lower limit logic (LLL), a set of abnormalities and an adaptive strategy. The LLL must be reflexive, compact and transitive. ${ }^{8}$ In our case the lower limit logic is PMDL. The idea is that the resulting adaptive logics strengthen PMDL by considering abnormalities as false 'as much as possible', where the latter phrase is disambiguated by the adaptive strategy. Proof-theoretically, we realize this idea by (a) allowing all PMDL-inferences to remain valid in an adaptive proof and (b) allowing for some extra inferences by means of a (PMDL-invalid) conditional rule.

Let us explicate point (b) by means of some examples. Reconsider (47)-(49). Although these inferences are PMDL-invalid, the following do hold in PMDL:

$$
\mathrm{O} \square_{J}(\neg A \vee B), \mathrm{O} \square_{J} A \vdash \mathbf{P M D L} \mathrm{O} \square_{J} B \vee \mathrm{P} \diamond_{J}(A \wedge \neg A)
$$

\footnotetext{
${ }^{8}$ In the literature on adaptive logics it is often required that the classical connectives are superimposed on the language of the lower limit logic in order to guarantee some metatheoretic properties. PMDL can be easily adjusted in that way. However, for the sake of presentation we omit this technical complication here.
} 


$$
\begin{gathered}
\mathrm{O} \neg A, \mathrm{O}(A \vee B) \vdash_{\mathrm{PMDL}} \mathrm{O} B \vee \mathrm{P}(A \wedge \neg A) \\
\square_{J} A, \square_{J}(\neg A \vee B) \vdash_{\mathbf{P M D L}} \square_{J} B \vee \diamond_{J}(A \wedge \neg A)
\end{gathered}
$$

Moreover, PMDL allows for the following 'weak' variants of Modus Ponens and Disjunctive Syllogism:

$$
\begin{array}{r}
A, \neg A \vee B \vdash \text { PMDL } B \vee(A \wedge \neg A) \\
A, A \supset B \vdash \text { PMDL } B \vee(A \wedge \neg A)
\end{array}
$$

Whereas (47)-(51) all fail for PMDL, their weaker versions (52)-(56) are PMDLvalid. In all of these 'weakened' cases, the discussed inferences hold in PMDL in disjunction with a formula that expresses some counterintuitive consequence. For (52), this is the formula $\mathrm{P} \diamond_{J}(A \wedge \neg A)$, expressing that it is permitted that the inconsistency $A \wedge \neg A$ is consistent with $J$ 's actions. For (53), it is the formula $\mathrm{P}(A \wedge \neg A)$, expressing that the inconsistency $A \wedge \neg A$ is permitted. For (54), the counterintuitive alternative is the formula $\diamond_{J}(A \wedge \neg A)$, expressing that the inconsistency $A \wedge \neg A$ is consistent with $J$ 's actions. For (55) and (56), it is the plain contradiction $A \wedge \neg A$. What all these counterintuitive disjuncts have in common, is that, semantically, they entail that a contradiction is verified at some PMDL-accessible world.

The idea behind the conditional inference rule mentioned above in point (b) will be that we allow for the derivation of the left-hand disjuncts of the formulas derived in (52)-(56) on the assumption that the 'unwanted' right-hand disjuncts are not the case. In order to represent this assumption, each line $l$ in an adaptive proof is equipped with a column that features a set of formulas $\Delta$ that represent the assumptions that are made in order to derive the formula on line $l$. This set $\Delta$ is the condition of line $l$. Conditions are subsets of a specific set of formulas: so-called abnormalities. For all adaptive logics, the set $\Omega$ of abnormalities is a set of LLL-contingent formulas characterized by one or more logical forms $\mathcal{F}$. For the adaptive logics $\mathbf{P} \mathbf{M D L} \mathbf{r}^{\mathbf{r}}$ and $\mathbf{P} \mathbf{M D L} \mathbf{m}^{\mathbf{m}}$, $\Omega=\left\{\diamond_{1} \ldots \diamond_{n}(A \wedge \neg A) \mid A \in \mathcal{P}, \diamond_{i} \in\{\mathrm{P}\} \cup\left\{\diamond_{J} \mid J \subseteq_{\varnothing} I\right\}\right\}$. Intuitively, $\Omega$ is the set each member of which verifies an inconsistency in some accessible world in the PMDL-semantics. ${ }^{9,10}$

\footnotetext{
${ }^{9}$ It is allowed that $i=0$. Hence $A \wedge \neg A \in \Omega$ whenever $A \in \mathcal{P}$.

${ }^{10}$ In fact, members of $\Omega$ even cause contradictions to be verified at the actual world, since e.g. $\mathrm{P}(p \wedge \neg p) \vdash \mathbf{P M D L} \mathrm{O}(p \vee \neg p) \wedge \neg \mathrm{O}(p \vee \neg p), \mathrm{P} \diamond{ }_{J}(p \wedge \neg p) \vdash \mathbf{P M D L} \mathrm{O}_{J}(p \vee \neg p) \wedge \neg \mathrm{O} \square_{J}(p \vee \neg p)$, etc. Since every normative conflict gives rise to a member of $\Omega$, this is at odds with Priest's analysis of conflicting norms [34, Ch. 13]. Although Priest accepts the possibility of contradicting norms, he does not accept that OO-conflicts entail outright contradictions.

Readers dissatisfied with this analysis may safely drop the rules $\left(\mathrm{RO}_{\neg}\right)$ and $\left(\mathrm{RP}_{\neg}\right)$ in the syntactic characterization of PMDL. This blocks the derivation of contradictions from OOand OP-conflicts (of course, the PMDL-semantics can be adjusted accordingly).

Call the resulting logic $\mathbf{P M D L} \mathbf{L}_{*}$. Then $\mathrm{O} A \wedge \mathrm{O} \neg A \vdash \mathbf{P M D L} \mathrm{P} A \wedge \neg \mathrm{P} A$ and $\mathrm{O} A \wedge \mathrm{P} \neg A \vdash \mathbf{P M D L}$ $\mathrm{O} A \wedge \neg \mathrm{O} A$, whereas $\mathrm{O} A \wedge \mathrm{O} \neg A \forall \mathbf{P M D L}_{*} \mathrm{P} A \wedge \neg \mathrm{P} A$ and $\mathrm{O} A \wedge \mathrm{P} \neg A \forall \mathbf{P M D L}_{*} \mathrm{O} A \wedge \neg \mathrm{O} A$.

Analogously to $\mathbf{P M D L}, \mathbf{P} \mathbf{M D L} *$ can be extended adaptively (with $\Omega$ defined as before). In the resulting extensions $\mathbf{P M D L}_{*}^{\mathbf{r}}$ and $\mathbf{P M D L}_{*}^{\mathbf{m}}$, the rules $(\mathrm{RO} \neg)$ and $\left(\mathrm{RP}_{\neg}\right)$ are applicable 'as much as possible', since e.g. $\mathrm{O} \neg A \vdash \mathbf{P M D L}_{*} \neg \mathrm{P} A \vee \mathrm{P}(A \wedge \neg A)$ and $\mathrm{P} \neg A \vdash \mathbf{P M D L}_{*} \neg \mathrm{O} A \vee$ $\mathrm{P}(A \wedge \neg A)$.
} 
Since our aim is to interpret a given set of premises as consistently as possible the set $\Omega$ is defined in such a way that each normative conflict gives rise to a (disjunction of) abnormalities in PMDL. This is illustrated in the following table. Let $A \in \mathcal{P}$ :

$$
\begin{aligned}
& \mathrm{O} \square_{J} A \wedge \mathrm{O} \square_{K} \neg A \vdash_{\text {PMDL }} \mathrm{P}(A \wedge \neg A) \\
& \mathrm{O} \square_{J} A \wedge \mathrm{P} \square_{K} \neg A \vdash_{\text {PMDL }} \mathrm{P}(A \wedge \neg A) \\
& \mathrm{O} \square_{J} A \wedge \mathrm{O} \square_{J} \neg A \vdash_{\text {PMDL }} \mathrm{P} \diamond_{J}(A \wedge \neg A) \\
& \mathrm{O} \square_{J} A \wedge \mathrm{P} \square_{J} \neg A \vdash_{\text {PMDL }} \mathrm{P} \diamond_{J}(A \wedge \neg A) \\
& \mathrm{O} \square_{J} A \wedge \mathrm{O} \neg \square_{J} A \vdash_{\text {PMDL }} \mathrm{P} \diamond_{J}(A \wedge \neg A) \\
& \mathrm{O} \square_{J} A \wedge \mathrm{P} \neg \square_{J} A \vdash_{\text {PMDL }} \mathrm{P} \diamond_{J}(A \wedge \neg A) \\
& \mathrm{O} A \wedge \mathrm{O} \neg A \vdash_{\text {PMDL }} \mathrm{P}(A \wedge \neg A) \\
& \mathrm{O} A \wedge \mathrm{P} \neg A \vdash_{\text {PMDL }} \mathrm{P}(A \wedge \neg A)
\end{aligned}
$$

Where $A \notin \mathcal{P}$, it is easy to see that due to the validity of de Morgan's laws the inferences in this table can be generalized to conflicts between more complex formulas. These will give rise to disjunctions of abnormalities. Let for instance $A=A_{1} \vee A_{2}$. Then, for example:

$$
\mathrm{O} \square_{J} A, \mathrm{O} \square_{K} \neg A \vdash \mathrm{PMDL} \mathrm{P}\left(A_{1} \wedge \neg A_{1}\right) \vee \mathrm{P}\left(A_{2} \wedge \neg A_{2}\right)
$$

If $A_{1}, A_{2} \in \mathcal{P}$, then $\mathrm{P}\left(A_{1} \wedge \neg A_{1}\right), \mathrm{P}\left(A_{2} \wedge \neg A_{2}\right) \in \Omega$. Otherwise, $\mathrm{P}\left(A_{1} \wedge \neg A_{1}\right) \vee$ $\mathrm{P}\left(A_{2} \wedge \neg A_{2}\right)$ can be further analyzed into a (longer) disjunction of abnormalities.

Adaptive logics take abnormalities to be false as long as there is no good reason to assume otherwise. This idea is made technically precise by means of two mechanisms: (i) a generic conditional rule $\mathrm{RC}$ that makes it possible to derive formulas conditionally and (ii) an adaptive strategy with a corresponding marking definition which determines when the assumption specified in the condition of a line is violated. If such a violation occurs, the condition is 'marked' and the derivation is disabled in the proof.

Let $\Gamma$ be a set of premises, and let

\section{$A \quad \Delta$}

abbreviate that $A$ occurs in the proof on the condition $\Delta$. Where $\Theta \subseteq \Omega$, the generic conditional rule $\mathrm{RC}$ is defined as follows:

$$
\begin{array}{ll}
\mathrm{RC} \quad \text { If } A_{1}, \ldots, A_{n} \vdash \mathbf{P M D L} B \vee \vee \Theta & A_{1} \Delta_{1} \\
\vdots & \vdots \\
& A_{n} \Delta_{n} \\
\hline B \quad & \Delta_{1} \cup \ldots \cup \Delta_{n} \cup \Theta
\end{array}
$$

Applied to (52)-(56), RC allows us to derive from the premises the lefthand disjunct on the assumption that the right-hand disjunct is false. E.g. for (52), we can derive $\mathrm{O} \square_{J} B$ from $\mathrm{O} \square_{J}(\neg A \vee B)$ and $\mathrm{O} \square_{J} A$ on the condition $\{\mathrm{P} \diamond(A \wedge \neg A)\}$. The same reasoning applies to (53)-(56). 
Next to RC, adaptive proofs make use of two other - less complicated generic rules: the premise introduction rule PREM, and the unconditional rule RU:

PREM If $A \in \Gamma$ :

\begin{tabular}{cc}
$\vdots$ & $\vdots$ \\
\hline$A \quad \varnothing$
\end{tabular}

RU

$$
\begin{aligned}
& \text { If } A_{1}, \ldots, A_{n} \vdash \text { PMDL } B: \quad A_{1} \quad \Delta_{1} \\
& \vdots \quad \vdots \\
& \begin{array}{ll}
A_{n} & \Delta_{n} \\
\hline B & \Delta_{1} \cup \ldots \cup \Delta_{n}
\end{array}
\end{aligned}
$$

PREM allows to introduce premises on the empty condition. RU gives the adaptive logic all the power of its LLL: whenever $B$ is PMDL-derivable from $A_{1}, \ldots, A_{n}$ it is also derivable in the adaptive logic. As for the conditional rule $\mathrm{RC}$, the conditions on which the $A_{i}$ 's are derived are carried forward to the line on which $B$ is derived. Note, however, that - just like PREM - RU does not allow us to introduce new conditions in an adaptive proof.

Let us take a look at a concrete example. Suppose that we have the premises $\mathrm{O} \square_{J} \neg p$ and $\mathrm{O} \square_{J}(p \vee q)$. We enter the premises via the premise introduction rule:

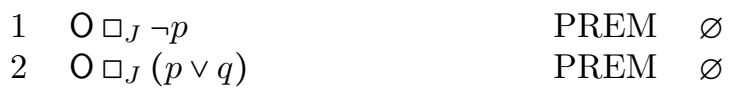

Given (52), we can apply RC to the premises at lines 1 and 2 and derive $\mathrm{O} \square_{J} q$ on the condition $\left\{\mathrm{P} \diamond_{J}(p \wedge \neg p)\right\}$ :

$$
3 \quad \mathrm{O} \square_{J} q \quad 1,2 ; \mathrm{RC} \quad\left\{\mathrm{P} \diamond_{J}(p \wedge \neg p)\right\}
$$

Now suppose that we introduce a new premise $\mathrm{P} \square_{J} p$ at line 4 :

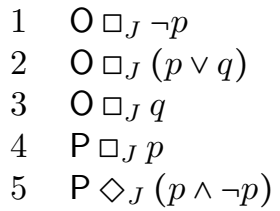

$\begin{array}{ll}\text { PREM } & \varnothing \\ \text { PREM } & \varnothing \\ 1,2 ; \text { RC } & \left\{\mathrm{P} \diamond_{J}(p \wedge \neg p)\right\} \vee^{5} \\ \text { PREM } & \varnothing \\ 1,4 ; \text { RU } & \varnothing\end{array}$

In view of $(60)$, we can use $\mathrm{RU}$ in order to derive $\mathrm{P} \diamond_{J}(p \wedge \neg p)$ from the formulas at lines 1 and 4 . Since the conditions of these lines are empty, the condition of line 5 is empty too. At line 5 we have derived the condition of line 3. Thus, the assumption made at line 3 that $\mathrm{P} \diamond_{J}(p \wedge \neg p)$ is false can no longer be upheld at stage 5 of the proof. Therefore we mark line 3 with a " $\checkmark$ " sign to denote that the formula at this line is no longer considered derived. This marking mechanism is governed by a marking definition, which differs depending on the adaptive strategy used. Before we can introduce the marking definitions for the different strategies however, some more terminology is required. 


\subsubsection{Two strategies}

At the beginning of Section 5.1.1 we mentioned that adaptive logics are characterized by a LLL, a set of abnormalities, and an adaptive strategy. The logics $\mathbf{P M D L}^{\mathbf{r}}$ and $\mathbf{P}$ MDL ${ }^{\mathbf{m}}$ both have PMDL as their LLL and $\Omega$ as their set of abnormalities. They differ only in the adaptive strategy they employ. Whereas $\mathbf{P M D L}^{\mathbf{r}}$ uses the reliability strategy, $\mathbf{P} \mathbf{M D L}{ }^{\mathbf{m}}$ uses the minimal abnormality strategy. Proof-theoretically, the strategy is governed by a marking mechanism as mentioned above. Each strategy makes use of a different marking definition which takes care of the markings in adaptive proofs. We first define the marking definition for the reliability strategy. Next, we turn to the marking definition for minimal abnormality. We illustrate the difference between both definitions by means of an example.

Where $\Delta$ is a non-empty finite set of abnormalities we write $\operatorname{Dab}(\Delta)$ instead of $\bigvee \Delta . \operatorname{Dab}(\Delta)$ is called a disjunction of abnormalities, or Dab-formula. In case $\operatorname{Dab}(\Delta)$ is derived at some stage $s$ of the proof on the condition $\varnothing$ and for no $\Delta^{\prime} \subset \Delta, \operatorname{Dab}\left(\Delta^{\prime}\right)$ has been derived at stage $s$ on the condition $\varnothing$, then $\operatorname{Dab}(\Delta)$ is a minimal Dab-formula at stage $s$. Where $\Delta_{1}, \Delta_{2}, \ldots$ are the minimal $D a b$-formulas at stage $s$ derived from $\Gamma$, we define the set of unreliable formulas at stage $s$ by $U_{s}(\Gamma)=\Delta_{1} \cup \Delta_{2} \cup \ldots$. The idea behind the marking definition for the reliability strategy is to mark lines whose condition contains unreliable formulas.

Definition 1 (Marking for reliability). Line $i$ with condition $\Delta$ is marked at stage $s$ iff $U_{s}(\Gamma) \cap \Delta \neq \varnothing$.

Let $\Gamma=\left\{\mathrm{O} \square_{J}(p \vee r), \mathrm{O} \square_{J}(q \vee r), \mathrm{O} \square_{J}(\neg p \wedge \neg q), \mathrm{P} \square_{J}(p \vee q)\right\}$. We start a $\mathbf{P M D L}^{\mathbf{r}}$-proof from $\Gamma$ by entering the premises:

$\begin{array}{llll}1 & \mathrm{O} \square_{J}(p \vee r) & \text { PREM } & \varnothing \\ 2 & \mathrm{O} \square_{J}(q \vee r) & \text { PREM } & \varnothing \\ 3 & \mathrm{O} \square_{J}(\neg p \wedge \neg q) & \text { PREM } & \varnothing \\ 4 & \mathrm{P} \square_{J}(p \vee q) & \text { PREM } & \varnothing\end{array}$

Since $\mathrm{O} \square_{J}(p \vee r), \mathrm{O} \square_{J}(\neg p \wedge \neg q) \vdash \mathbf{P M D L} \mathrm{O} \square_{J} r \vee \mathrm{P} \diamond_{J}(p \wedge \neg p)$, we can apply $\mathrm{RC}$ to lines 1 and 3 and conditionally derive $\mathrm{O} \square_{J} r$ at line 5 :

$$
\begin{array}{llll}
5 & \mathrm{O} \square_{J} r & 1,3 ; \mathrm{RC} & \left\{\mathrm{P} \diamond_{J}(p \wedge \neg p)\right\} \vee^{6} \\
6 & \mathrm{P} \diamond_{J}(p \wedge \neg p) \vee \mathrm{P} \diamond_{J}(q \wedge \neg q) & 3,4 ; \mathrm{RU} & \varnothing
\end{array}
$$

At stage 6 of the proof, $U_{6}(\Gamma)=\left\{\mathrm{P} \diamond_{J}(p \wedge \neg p), \mathrm{P} \diamond_{J}(q \wedge \neg q)\right\}$ in view of the $D a b$-formula derived at line 6 . Since the condition of line 5 intersects with $U_{6}(\Gamma)$, line 5 is marked at stage 6 in view of Definition 1 .

Reliability is a very cautious strategy. As soon as an abnormality occurs in some minimal $D a b$-formula derived in a proof, all inferences that rely on the falsity of this abnormality are marked. In the example above, $J$ 's duty to bring about $r$ is not $\mathbf{P M D L} \mathbf{L}^{\mathbf{r}}$-derivable since it cannot be guaranteed that $p$ behaves consistently in all accessible worlds. However, in this example $J$ can consistently satisfy all of his/her/its duties by bringing about $\neg p, \neg q$, and $r$. Hence, we might 
want to apply a different strategy - the minimal abnormality strategy - that allows for some slightly 'bolder' inferences.

Suppose that the proof from $\Gamma$ is a $\mathbf{P M D L} \mathbf{m}^{\mathbf{m}}$-proof, and that we continue it as follows (we repeat the proof from line 5 onwards):

$$
\begin{array}{llll}
5 & \mathrm{O} \square_{J} r & 1,3 ; \mathrm{RC} & \left\{\mathrm{P} \diamond_{J}(p \wedge \neg p)\right\} \\
6 & \mathrm{P} \diamond_{J}(p \wedge \neg p) \vee \mathrm{P} \diamond_{J}(q \wedge \neg q) & 3,4 ; \mathrm{RU} & \varnothing \\
7 & \mathrm{O} \square_{J} r & 2,3 ; \mathrm{RC} & \left\{\mathrm{P} \diamond_{J}(q \wedge \neg q)\right\}
\end{array}
$$

Note that, if this were a $\mathbf{P M D L}{ }^{\mathbf{r}}$-proof, lines 5 and 7 would be marked at stage 7 of the proof. Not so for $\mathbf{P M D L}^{\mathbf{m}}$ however. The minimal abnormality strategy is 'bolder' than reliability in that it assumes that, when a minimal $D a b$-formula has been derived, only one of the disjuncts of this formula need be true. Since in the example $\mathrm{O} \square_{J} r$ is derivable on the condition $\left\{\mathrm{P} \diamond_{J}(p \wedge \neg p)\right\}$ and on the condition $\left\{\mathrm{P} \diamond_{J}(p \wedge \neg p)\right\}$, and since only one of these abnormalities needs to be true in order to verify the $D a b$-formula derived at line 6 , we can still safely assume that the other abnormality is false.

Technically, this is realized as follows. A choice set of $\Sigma=\left\{\Delta_{1}, \Delta_{2}, \ldots\right\}$ is a set that contains one element out of each member of $\Sigma$. A minimal choice set of $\Sigma$ is a choice set of $\Sigma$ of which no proper subset is a choice set of $\Sigma$. Where $\operatorname{Dab}\left(\Delta_{1}\right), \operatorname{Dab}\left(\Delta_{2}\right), \ldots$ are the minimal $D a b$-formulas that are derived at stage $s, \Phi_{s}(\Gamma)$ is the set of minimal choice sets of $\left\{\Delta_{1}, \Delta_{2}, \ldots\right\}$.

Definition 2 (Marking for minimal abnormality). Where $A \in \mathcal{L}_{\text {MDL }}$ is derived at line $i$ of a proof from $\Gamma$ on a condition $\Delta$, line $i$ is marked at stage $s$ iff

(i) there is no $\Delta^{\prime} \in \Phi_{s}(\Gamma)$ such that $\Delta^{\prime} \cap \Delta=\varnothing$, or

(ii) for some $\Delta^{\prime} \in \Phi_{s}(\Gamma)$, there is no line at which $A$ is derived on a condition $\Theta$ for which $\Delta^{\prime} \cap \Theta=\varnothing$.

The reader can easily verify that - in view of Definition 2 - lines 5 and 7 of the proof remain unmarked at stage 7 .

Note that - whichever strategy is used - markings may come and go in adaptive proofs. A line may be marked at some stage $s$ of the proof, and unmarked again at a later stage $s^{\prime}$. Before we can define a consequence relation for the logics $\mathbf{P} \mathbf{M D L} \mathbf{L}^{\mathbf{r}}$ and $\mathbf{P} \mathbf{M D L} \mathbf{m}^{\mathbf{m}}$, we need a stable criterion for derivability: ${ }^{11}$

Definition 3. $A$ is finally derived from $\Gamma$ at line $i$ of a proof at finite stage $s$ iff (i) line $i$ is not marked at stage $s$, and (ii) every extension of the proof in which line $i$ is marked may be further extended in such a way that line $i$ is unmarked.

Where a $\mathbf{P M D L} \mathbf{r}^{\mathbf{r}}$-proof makes use of Definition 1 and a $\mathbf{P} \mathbf{M D L} \mathbf{m}^{\mathbf{m}}$-proof makes use of Definition 2, consequence relations for $\mathbf{P} \mathbf{M D L} \mathbf{L}^{\mathbf{r}}$ and $\mathbf{P} \mathbf{M D L} \mathbf{L}^{\mathbf{m}}$ are defined as follows:

Definition 4. $\Gamma \vdash{ }_{\mathbf{P M D L}} \mathbf{r} A$ ( $A$ is finally $\mathbf{P} \mathbf{M D L} \mathbf{L}^{\mathbf{r}}$-derivable from $\Gamma$ ) iff there is a $\mathbf{P M D L} \mathbf{L}^{\mathbf{r}}$-proof in which $A$ is finally derived from $\Gamma$ at some line of a proof from $\Gamma$.

\footnotetext{
${ }^{11}$ In [4], this Definition 3 is interpreted as a two-player game in which the proponent has a winning strategy in case she has a reply to every counterargument by her opponent.
} 
Definition 5. $\Gamma \vdash$ PMDL $^{\mathbf{m}} A$ ( $A$ is finally $\mathbf{P} \mathbf{M D L}{ }^{\mathbf{m}}$-derivable from $\Gamma$ ) iff there is a $\mathbf{P M D L}^{\mathbf{m}}$-proof in which $A$ is finally derived from $\Gamma$ at some line of a proof from $\Gamma$.

Thus, where $\Gamma=\left\{\mathrm{O} \square_{J}(p \vee r), \mathrm{O} \square_{J}(q \vee r), \mathrm{O} \square_{J}(\neg p \wedge \neg q), \mathrm{P} \square_{J}(p \vee q)\right\}$, $\Gamma \vdash \mathbf{P M D L}^{\mathrm{r}} \mathrm{O} \square_{J} r$, yet $\Gamma \vdash \mathbf{P M D L}^{\mathrm{m}} \mathrm{O} \square_{J} r$.

Here are some more illustrations of the proof theory for $\mathbf{P} \mathbf{M D L} \mathbf{L}^{\mathbf{r}}$ and $\mathbf{P} \mathbf{M D L} \mathbf{m}^{\mathbf{m}}$. In the remainder, we write $\mathbf{P} \mathbf{M D L}{ }^{\mathbf{x}}$ as a generic name for both $\operatorname{logics}(\mathbf{x} \epsilon$ $\{r, m\})$.

Example 1. Let $\Gamma_{1}=\left\{\mathrm{O} \square_{J} \mathrm{O} \square_{K} p, \mathrm{O} \square_{J} \mathrm{O} \square_{K}(\neg p \vee q), \mathrm{O} \square_{J} r, \mathrm{P} \square_{K} \neg r\right\}$. We start a $\mathbf{P}$ MDL ${ }^{\mathrm{x}}$-proof from $\Gamma_{1}$ by entering the premises:

$\begin{array}{llll}1 & \mathrm{O} \square_{J} \mathrm{O} \square_{K} p & \text { PREM } & \varnothing \\ 2 & \mathrm{O} \square_{J} \mathrm{O} \square_{K}(\neg p \vee q) & \text { PREM } & \varnothing \\ 3 & \mathrm{O} \square_{J} r & \text { PREM } \varnothing \\ 4 & \mathrm{P} \square_{K} \neg r & \text { PREM } & \varnothing \\ 5 & \mathrm{P}(r \wedge \neg r) & 3,4 ; \mathrm{RU} & \varnothing \\ 6 & \mathrm{O} \square_{J} \mathrm{O} \square_{K} q & 1,2 ; \mathrm{RC} \quad\left\{\mathrm{P} \diamond_{J} \mathrm{P} \diamond_{K}(p \wedge \neg p)\right\}\end{array}$

In view of Definitions 4 and $5, \Gamma_{1} \vdash \mathbf{P M D L}^{\times} \mathrm{O} \square_{J} \mathrm{O} \square_{K} q$.

Example 2. Let $\Gamma_{2}=\left\{\mathrm{O}\left(\neg \square_{K} p \supset \square_{J} p\right), \mathrm{O} \square_{K} \neg \square_{K} p\right\}$. We start a $\mathbf{P M D L} \mathbf{L}^{\mathbf{x}}$-proof from $\Gamma_{2}$ by entering the premises:

$\begin{array}{llll}1 & \mathrm{O}\left(\neg \square_{K} p \supset \square_{J} p\right) & \text { PREM } & \varnothing \\ 2 & \mathrm{O} \square_{K} \neg \square_{K} p & \text { PREM } & \varnothing \\ 3 & \mathrm{O} \square_{J} p & 1,2 ; \mathrm{RC} & \left\{\mathrm{P} \diamond_{K}(p \wedge \neg p)\right\}\end{array}$

In view of Definitions 4 and $5, \Gamma_{2} \vdash \mathbf{P M D L}^{\times} \mathrm{O} \square_{J} p$.

\subsection{Semantics and meta-theory for $\mathrm{PMDL}^{\mathrm{r}}$ and $\mathrm{PMDL}^{\mathrm{m}}$}

Where $\mathbf{x} \in\{r, m\}$, the $\mathbf{P} \mathbf{M D L} \mathbf{x}^{\mathbf{x}}$-semantics proceeds in the style of Shoham (see $[37, ?])$ by selecting a certain subset of the PMDL-models. Let $A b(M)$, the abnormal part of the model $M$, abbreviate the set of abnormalities verified by $M$, i.e. $A b(M)=\{A \in \Omega \mid M \Vdash \mathbf{P M D L} A\}$. The minimal abnormality strategy selects all PMDL-models of a premise set $\Gamma$ which have a minimal abnormal part (with respect to set-inclusion):

Definition 6. A PMDL-model $M$ of $\Gamma$ is minimally abnormal iff there is no PMDL-model $M^{\prime}$ of $\Gamma$ such that $A b\left(M^{\prime}\right) \subset A b(M)$.

The semantic consequence relation for $\mathbf{P} \mathbf{M D L} \mathbf{m}^{\mathbf{m}}$ is defined by selecting all minimally abnormal PMDL-models:

Definition 7. $\Gamma \Vdash_{\mathbf{P M D L}^{\mathrm{m}}} A$ iff $A$ is verified by all minimally abnormal PMDLmodels.

Before we can define the semantic consequence relation for $\mathbf{P M D L}{ }^{\mathbf{r}}$, we need some more terminology. We say that a $D a b$-formula $D a b(\Delta)$ is a $D a b$ consequence of $\Gamma$ if it is PMDL-derivable from $\Gamma$; it is a minimal Dab-consequence 
of $\Gamma$ if there is no $\Delta^{\prime} \subset \Delta$ such that $\operatorname{Dab}\left(\Delta^{\prime}\right)$ is a $D a b$-consequence of $\Gamma$. The set of formulas that are unreliable with respect to $\Gamma$, denoted by $U(\Gamma)$, is defined by:

Definition 8. Where $\operatorname{Dab}\left(\Delta_{1}\right), \operatorname{Dab}\left(\Delta_{2}\right), \ldots$ are the minimal $D a b$-consequences of $\Gamma, U(\Gamma)=\Delta_{1} \cup \Delta_{2} \cup \ldots$ is the set of formulas that are unreliable with respect to $\Gamma$.

Definition 9. A PMDL-model $M$ of $\Gamma$ is reliable iff $A b(M) \subseteq U(\Gamma)$.

The semantic consequence relation for $\mathbf{P} \mathbf{M D L} \mathbf{L}^{\mathbf{r}}$ is defined by selecting all reliable PMDL-models:

Definition 10. $\Gamma \Vdash$ PMDL $^{\mathbf{r}} A$ iff $A$ is verified by all reliable models of $\Gamma$.

For any adaptive logic in standard format which has a LLL that is sound and complete with respect to its semantics, there is a generic proof that the adaptive logic is sound and complete with respect to its semantics:

Theorem 2. $\Gamma \vdash_{\mathbf{P M D L}^{\mathbf{r}}} A$ iff $\Gamma \Vdash{ }_{\mathbf{P M D L}} \mathbf{r} A$.

$\Gamma \vdash \mathbf{P M D L}^{\mathrm{m}} A$ iff $\Gamma \Vdash \mathbf{P M D L}^{\mathrm{m}} A$.

For $\mathbf{P M D L}^{\mathbf{r}}$, the proof of Theorem 2 follows immediately from Theorem 1 from Section 4.2 and Corollary 2 from [3]. For $\mathbf{P M D L}^{\mathbf{m}}$, the proof follows immediately from Theorem 1 from Section 4.2 and Theorem 9 from [3].

The fact that the set of $\mathbf{P} \mathbf{M D L} \mathbf{L}^{\mathbf{x}}$-models of $\Gamma$ is a subset of the set of PMDL-models of $\Gamma$ immediately ensures that $\mathbf{P} \mathbf{M D L}^{\mathrm{x}}$ strengthens PMDL.

Theorem 3. If $\Gamma \Vdash$ PMDL $A$, then $\Gamma \Vdash$ PMDL $^{\times} A$.

Where $\mathcal{M}_{\Gamma}^{\mathrm{PMDL}}, \mathcal{M}_{\Gamma}^{m}$, and $\mathcal{M}_{\Gamma}^{r}$ denote the set of PMDL-models, minimally abnormal PMDL-models, resp. reliable PMDL-models of $\Gamma$, we also know that:

Theorem 4. (Strong Reassurance.) If $M \in \mathcal{M}_{\Gamma}^{\mathrm{PMDL}}-\mathcal{M}_{\Gamma}^{m}$, then there is a $M^{\prime} \in \mathcal{M}_{\Gamma}^{m}$ such that $A b\left(M^{\prime}\right) \subset A b(M)$. If $M \in \mathcal{M}_{\Gamma}^{\mathrm{PMDL}}-\mathcal{M}_{\Gamma}^{r}$, then there is a $M^{\prime} \in \mathcal{M}_{\Gamma}^{r}$ such that $A b\left(M^{\prime}\right) \subset A b(M)$.

Theorem 4 is shown generically for adaptive logics in standard format as Corollary 1 in [3].

In case no $D a b$-formulas are derivable from a premise set by means of the LLL, it is safe to consider all abnormalities as false. As a consequence, the adaptive logic will then yield the same consequence set as the logic that interprets all abnormalities as false (or equivalently, as the logic that fully validates the inference rules whose application the adaptive logic only allows conditionally). This logic is called the upper limit logic of an adaptive logic. The upper limit logic of an adaptive logic is obtained by adding to its LLL one or more axioms and/or rules that trivialize exactly those formulas that are members of $\Omega$. The upper limit logic $\mathbf{U L L}$ of $\mathbf{P} \mathbf{M D L} \mathbf{L}^{\mathbf{x}}$ is defined by adding to PMDL the rule (ECQ). ULL is related to PMDL as set out by the Derivability Adjustment Theorem: 
Theorem 5. $\Gamma \vdash_{\mathbf{U L L}} A$ iff (there is $a \Delta \subseteq \Omega$ for which $\Gamma \vdash_{\mathbf{P M D L}} A \vee \operatorname{Dab}(\Delta)$ or $\Gamma \vdash$ PMDL $A$ ).

The set of $D a b$-consequences derivable from the premise set determines the amount to which the consequence set of $\mathbf{P} \mathbf{M D L}^{\mathbf{x}}$ resembles the ULLconsequence set. This is why adaptive logicians say that $\mathbf{P M D L} \mathbf{L}^{\mathbf{x}}$ adapts itself to a premise set. $\mathbf{P} \mathbf{M D L}{ }^{\mathbf{x}}$ is always at least as strong as $\mathbf{P M D L}$ and maximally as strong as ULL:

Theorem 6. $C n_{\mathrm{PMDL}}(\Gamma) \subseteq C n_{\mathbf{P M D L}^{\times}}(\Gamma) \subseteq C n_{\mathrm{ULL}}(\Gamma)$.

In view of Theorem 11 from [3], it follows immediately that:

Corollary 1. $C n_{\mathbf{P M D L}}(\Gamma) \subseteq C n_{\mathbf{P M D L}^{\mathbf{r}}}(\Gamma) \subseteq C n_{\mathbf{P M D L}^{\mathrm{m}}}(\Gamma) \subseteq C n_{\mathrm{ULL}}(\Gamma)$.

If $\Gamma$ is normal, i.e. if $U(\Gamma)=\varnothing$, then we can even prove a stronger result:

Theorem 7. If $\Gamma$ is normal, then $C n_{\mathbf{P M D L}^{x}}(\Gamma)=C n_{\mathbf{U L L}}(\Gamma)$.

For the proofs of Theorems 5-7, and for an overview of further meta-theoretic properties of adaptive logics, we refer to [3].

The reader may have noticed that ULL trivializes contradictions at accessible worlds, thus promoting " $\neg$ " to a fully classical negation connective. It should come as no surprise then, that ULL is just MDL in disguise:

Theorem 8. $\Gamma \vdash_{\mathrm{ULL}} A$ iff $\Gamma \vdash_{\mathrm{MDL}} A$.

A proof outline for Theorem 8 is contained in Section $\mathrm{C}$ of the Appendix.

Corollary 2. (i) $C n_{\mathbf{P M D L}}(\Gamma) \subseteq C n_{\mathbf{P M D L}^{\mathrm{r}}}(\Gamma) \subseteq C n_{\mathbf{P M D L}^{\mathrm{m}}}(\Gamma) \subseteq C n_{\mathrm{MDL}}(\Gamma)$. (ii) If $\Gamma$ is normal, then $C n_{\mathbf{P M D L}^{\mathbf{x}}}(\Gamma)=C n_{\mathbf{M D L}}(\Gamma)$.

(i) follows by Corollary 1 and Theorem 8, (ii) follows by Theorems 7 and 8 .

\section{Related work}

\subsection{Logics of action and stit-logic}

The logics presented in this paper are not defined within either of the two 'main' paradigms for representing actions in (deontic) logic, i.e. stit-logic [8, 9, 22, 26] and dynamic logic $[10,31]$. Nonetheless, our $\square_{J}$ operators resemble in some respects the Chellas stit or cstit operators used in stit logic. In our framework, a formula $\square_{J} A$ is interpreted as " $J$ brings about $A$ ". In stit-logic, a formula $[J$ stit : $A]$ is interpreted as " $J$ sees to it that $A$ ". On both accounts, $A$ is a state of affairs, and not an action nominal as is the case in e.g. dynamic logic. Moreover, the notions of refrainment and deliberative agency as defined in Section 2.2 are analogous to those of stit logic.

A first major difference between the logics defined here and stit-logics is that the stit-framework is temporal/prospective, while we work in an atemporal 
setting. It is a question for future research to extend the framework given here with the ability to reason about future (and maybe past) states.

A second difference between both approaches is that the $\square_{J}$ operators defined here are $\mathbf{S 4 - m o d a l i t i e s , ~ w h i l e ~ c s t i t ~ o p e r a t o r s ~ - ~ t h e i r ~ a n a l o g u e s ~ i n ~ s t i t ~ l o g i c ~ - ~ a r e ~}$ S5-modalities. Thus, in MDL the $\left(5_{J}\right)$ schema is invalid:

$$
\diamond_{J} A \supset \square_{J} \diamond_{J} A
$$

Note that if $\left(5_{J}\right)$ were valid, then the 'Brouwerian' schema $\left(\mathrm{B}_{J}\right)$ too would hold for our agentive operators:

$$
A \supset \square_{J} \diamond_{J} A
$$

Intuitively, $\left(\mathrm{B}_{J}\right)$ requires that if $A$ is the case, then all agents guarantee that $A$ is consistent with their actions. This is a very strong requirement. If $A$ is indeed the case, then normally we try to act on this fact as much as possible. But there are exceptions. We might, for instance, not know that $A$ is the case, we might not be aware of it etc. In such cases, $A$ need not be consistent with our actions. Therefore we opted to leave $\left(\mathrm{B}_{J}\right)$ (and, consequently, $\left(5_{J}\right)$ ) out of our axiomatization. ${ }^{12}$

A third difference worth pointing out is that our systems differ from stit logics in their treatment of collective actions and obligations. In stit logic, operators for agency are closed under 'weakening' by the addition of further agents: If $J \subset K \subseteq_{\varnothing} I$, then if $J$ sees to it that $A$, then $K$ sees to it that $A$. As illustrated in (5) and (6), this kind of weakening is invalid in the logics defined here. Consequently, a statement like (66) is ML-,MDL-,PMDL-, and $\mathbf{P M D L}^{\mathrm{x}}$-consistent, while its stit analogue would cause explosion: ${ }^{13}$

$$
\square_{i} A \wedge \square_{j} A \wedge \neg \square_{\{i, j\}} A
$$

Let us further illustrate this property by generalizing it to the deontic setting. Suppose that two agents $i$ and $j$ are divorced and that they work for the same company. Then we can imagine that, when faced with a certain task $A$, it makes sense for the boss $k$ to issue the following obligations:

$$
\square_{k}\left(\mathrm{O}\left(\square_{i} A \vee \square_{j} A\right) \wedge \neg \mathrm{P} \square_{\{i, j\}} A\right)
$$

Thus, one of $i$ and $j$ should bring about $A$, but they should not do it together (because since the divorce they are no longer on speaking terms).

Finally, the logics presented in this paper allow for the consistent formalization of other-agent nested agentive formulas of the form " $J$ brings it about that $K$ brings it about that $A$ " (i.e. $\square_{J} \square_{K} A$ ). In the stit-framework, this is

\footnotetext{
${ }^{12}$ A very welcome consequence of not having $\left(5_{J}\right)$ is that - as opposed to refrainment for the cstit-operator - refrainment for the $\square_{J}$-operator does not collapse into simple non-action: $\square_{J} \neg \square_{J} A \vdash_{\text {MDL }} \neg \square_{J} A$, but $\neg \square_{J} A \nvdash_{\text {MDL }} \square_{J} \neg \square_{J} A$. In stit theory, refraining is for this reason modelled in terms of the deliberative stit-operator (see [22, Sec. 2.3.3]).

${ }^{13}$ A notable exception here is the sstit or 'strictly sees to it that' operator for joint agency as defined in [8]. See also [9, pp. 284-287].
} 
impossible (see [9, Sec. 10B.1] for a proof of the impossibility of other-agent nested stits).

Altogether, these differences motivate our approach as a pursuit-worthy alternative for existing logics of action.

\subsection{Conflict-tolerant deontic logic}

SDL can be made more conflict-tolerant either by enriching its language, or by weakening its axioms and/or rules. In [26], Kooi and Tamminga deal with conflicting norms by enriching SDL so as to be capable of distinguishing between various sources and interest groups in view of which norms arise. Moreover, following [22] they equip their system with modal stit-operators for dealing with the difficult notion of (moral) agency. Similarly, we could try to deal with conflicting norms by imposing a preference ordering on our obligations and permissions, e.g. [20].

Such extensions are very successful in increasing the expressive power of SDL, but they are unable to consistently accommodate all normative conflicts. Conflicts may arise between norms promulgated at the same time, by the same authority. It is not difficult to see how we could extend this type of reasoning to norms of the same footing, addressed at the same group of people etc. so that in the end we need a logic that invalidates at least some SDL-theorems if we want to deal with all instances of normative conflicts.

$\mathbf{P M D L}^{\mathbf{r}}$ and $\mathbf{P} \mathbf{M D L}{ }^{\mathbf{m}}$ weaken SDL by dropping (ECQ). In a non-agentive setting, paraconsistent deontic logics were presented in $[6,7,11,34]$. Notwithstanding their representation of agents, the logics presented here are closely related to the system $\mathbf{D P}^{\mathbf{r}}$ from [7]. However, whereas $\mathbf{D P}^{\mathbf{r}}$ uses the paraconsistent logic $\mathbf{C L u N s}$ as its LLL, $\mathbf{P} \mathbf{M D L} \mathbf{r}^{\mathbf{r}}$ and $\mathbf{P} \mathbf{P D L}{ }^{\mathbf{m}}$ use an agentive extension of Priest's LP.

Next to the paraconsistent approach adopted here, other strategies can be adopted for weakening SDL. A popular strategy is to weaken the aggregation principle (ANDO). In a non-monotonic, non-agentive setting, this strategy was adopted in e.g. $[18,21,23,29,30]$. Another strategy open for accommodating normative conflicts is to weaken the inheritance principle (INHO). This approach was adopted in e.g. [14, 15, 39, 41].

\subsection{Adaptive deontic logic}

As mentioned in the introduction, this paper builds on earlier work on agentive adaptive deontic logics. More specifically, it continues the task set out in [6] of constructing a multi-agent adaptive deontic logic capable of tolerating normative conflicts. The system $\mathbf{P M D L}^{\mathbf{x}}$ improves on the semantics defined in [6] in various ways.

First, $\mathbf{P M D L} \mathbf{L}^{\mathbf{x}}$ is built 'on top' of the paraconsistent logic $\mathbf{L P}$, whereas the logic $\mathbf{M D P}{ }^{\mathbf{m}}$ from [6] is built 'on top' of the paraconsistent logic CLuNs from e.g. [2, 5]. The advantage of $\mathbf{L P}$ is that its implication is non-detachable and hence provides a better isolation of normative conflicts. For instance, from 
$\mathrm{O} A, \mathrm{O} \neg A$, and $\mathrm{O}(A \supset B)$ we cannot derive $\mathrm{O} B$ by means of $\mathbf{P} \mathbf{M D L} \mathbf{L}^{\mathbf{x}}$, but we can derive $\mathrm{O} B$ by means of $\mathbf{M D P}^{\mathrm{m}}$.

Second, the language of $\mathbf{P} \mathbf{M D L} \mathbf{L}^{\mathbf{x}}$ has no restrictions whatsoever on nested modal operators. This flexibility makes it easier to extend the language in various ways by adding extra modalities for representing e.g. knowledge, beliefs, commitments of agents and groups.

Third, as opposed to $\mathbf{M D P} \mathbf{P}^{\mathbf{m}}, \mathbf{P} \mathbf{M D L} \mathbf{L}^{\mathbf{x}}$ does not allow for distribution over disjunctive actions:

$$
\square_{J}(A \vee B){ }^{\mathbf{P M D L}^{\times}} \square_{J} A \vee \square_{J} B
$$

Suppose, for instance, that an agent flips a coin. In doing so she guarantees that either heads or tails will be the outcome, but she cannot determine the exact outcome of the flip. Hence she does not bring it about that heads is the outcome or bring it about that tails is the outcome.

Fourth, $\mathbf{P M D L}^{\mathbf{x}}$ is equipped with an adaptive proof theory, as illustrated in Section 5.1, whereas $\mathbf{M D P}^{\mathbf{m}}$ was only characterized semantically. Moreover, unlike $\mathbf{M D P} \mathbf{P}^{\mathbf{m}}, \mathbf{P M D L}^{\mathbf{x}}$ has a regular Kripke-semantics. Altogether, this makes $\mathbf{P} \mathbf{M D L}^{\mathbf{x}}$ the first sound and complete Kripke-style agentive adaptive (deontic) logic.

Most adaptive deontic logics interpret the given premises as non-conflicting as possible. However, they can also be applied in order to render defeasible other inferences and apply these "as much as possible". This has been realized for instance in the context of conditional obligations such as $\mathrm{O}(A \mid B): A$ is obligatory if $B$ is the case. In [40] factual detachment is defeasibly applied to $\mathrm{O}(A \mid B)$ and $B$ in order to derive the non-conditional obligation $\mathrm{O} A$. Similarly, in [39] strengthening the antecedent is applied to $\mathrm{O}(A \mid B)$ defeasibly in order to derive $\mathrm{O}(A \mid B \wedge C)$.

For some other adaptive deontic logics that can consistently accommodate instances of normative conflicts, see [7, 29, 39, 41]. For an introduction to the framework of adaptive logics, see [3].

\section{Conclusion and outlook}

We presented the logics $\mathbf{P M D L} \mathbf{L}^{\mathbf{r}}$ and $\mathbf{P} \mathbf{M D L} \mathbf{L}^{\mathbf{m}}$, two non-classical, non-monotonic logics for reasoning about conflicting collective actions and norms in a multiagent setting. These logics consistently allow for a broad variety of conflicts thanks to their paraconsistent negation connective. Due to their characterization within the adaptive logics framework for non-monotonic reasoning, the systems are sufficiently powerful to account for intuitive instances of rules like Modus Ponens, Disjunctive Syllogism and Contraposition - rules that are invalid in many monotonic paraconsistent logics. The adaptive proof theory of $\mathbf{P M D L}^{\mathbf{r}}$ and $\mathbf{P} \mathbf{M D L}{ }^{\mathbf{m}}$ explicates defeasible inference steps in a natural way and closely mirrors actual reasoning.

$\mathbf{P M D L}^{\mathbf{r}}$ and $\mathbf{P} \mathbf{M D L} \mathbf{m}^{\mathbf{m}}$ are the first agentive adaptive logics characterizable within a regular Kripke-style semantics. For this reason, they are well-suited for 
adding more expressivity to their language. We could, for instance, relativize the deontic operators in order to express by which authority various norms are issued. Moreover, it would be interesting to add epistemic modalities to the framework in order to express e.g. which agents and groups know each other's obligations. Future work also includes making the framework temporal, and perhaps adding to the language the power to express such notions as commitment or trust.

\section{Funding}

Research for this paper was supported by subventions from the Research Foundation - Flanders (FWO Vlaanderen), and from the Special Research Grant of Ghent University (BOF).

\section{Acknowledgments}

We thank the organizers and attendants of CLIMA XII and the anonymous referees for their valuable comments to our paper.

\section{APPENDIX}

\section{A The axiomatization of LP}

$\mathbf{L P}$ is axiomatized as follows:

$$
\begin{array}{ccc}
A, B \vdash A \wedge B & (\mathrm{AND}) & \neg A \vee \neg B \vdash \neg(A \wedge B) \\
A \wedge B \vdash A & (\mathrm{AN} 1) & \neg A \wedge \neg B \vdash \neg(A \vee B) \\
A \wedge B \vdash B & (\mathrm{AN} 2) & \neg(A \vee B) \vdash \neg A \wedge \neg B \\
A \vdash A \vee B & (\mathrm{OR} 1) & A \vdash \neg \neg A \\
B \vdash A \vee B & (\mathrm{OR} 2) & \neg \neg A \vdash A \\
\neg(A \wedge B) \vdash \neg A \vee \neg B & (\mathrm{DM} 1) & \vdash A \vee \neg A
\end{array}
$$

$$
\text { If } A, B \vdash D \text { and } A, C \vdash D \text {, then } A, B \vee C \vdash D \text {. }
$$

Moreover, the bottom constant $(\perp)$ is characterized by the following rule:

$$
\perp \vdash A
$$

Fact 1. (i) If $\Delta \cup\{A\} \vdash \mathbf{P M D L} C$ and $\Delta \cup\{B\} \vdash_{\mathbf{P M D L}} C$ then $\Delta \cup\{A \vee B\} \vdash_{\mathbf{P M D L}} C$.

(ii) The following rule holds in PMDL for each $J \subseteq_{\varnothing} I$ :

$$
\square_{J} A \vdash \diamond_{J} A
$$

It is easy to see that (i) follows by means of (RBC) and (ii) by means of $\left(\mathrm{T} \square_{J}\right)$ and $\left(\mathrm{T} \diamond_{J}\right)$. 


\section{B Soundness and completeness for PMDL}

As ML and MDL are fairly standard normal modal logics, we do not prove soundness and completeness theorems for these logics. Instead, we prove soundness and completeness for the more complex system PMDL.

Where $R \subseteq W \times W$ we use in the remainder the notation $R w=\left\{w^{\prime} \in W \mid R w w^{\prime}\right\}$.

Lemma 1. Where $M=\left\langle W,\left\langle R_{J}\right\rangle_{J \subseteq \varnothing I}, R_{\mathrm{O}}, v\right.$, @ $\rangle$ is a PMDL-model, we have: for all $w \in W$, if $M, w \neq A$ then $M, w \vDash \neg A$.

Proof. We show this by an induction over the length of $A$. Let $A \in \mathcal{P}$. Suppose $M, w \notin A$. By $\left(\mathrm{C}_{\neg}\right), M, w \vDash \neg A$.

For the induction step let first $A=B \wedge C$. Suppose $M, w \notin B \wedge C$. By $(\mathrm{C} \wedge)$, $M, w \notin B$ or $M, w \notin C$. By the induction hypothesis, $M, w \vDash \neg B$ or $M, w \vDash \neg C$. By $(\mathrm{C} \vee), M, w \vDash \neg B \vee \neg C$. By $(\mathrm{C} \neg \wedge), M, w \vDash \neg(B \wedge C)$. The cases $A=B \vee C$, and $A=\neg B$ are similar and left to the reader.

Let $A=\mathrm{O} B$. Suppose $M, w \neq \neg \mathrm{O} B$. By $(\mathrm{C} \neg \mathrm{O}) M, w \neq \mathrm{P} \neg B$. By $(\mathrm{CP})$ there is no $w^{\prime} \in R_{\mathrm{O}} w$ for which $M, w^{\prime} \vDash \neg B$. By the induction hypothesis, for all $w^{\prime} \in R_{\mathrm{O}} w$, $M, w^{\prime} \vDash \neg \neg B$ and hence by $\left(\mathrm{C}_{\neg \neg}\right), M, w^{\prime} \vDash B$. Thus, by (CO), $M, w \vDash \mathrm{O} B$.

The case $A=\square_{J} B$ is analogous and left to the reader.

Let $A=\mathrm{P} B$. Suppose $M, w \neq \mathrm{P} B$. Hence, by $(\mathrm{CP})$ there is no $w^{\prime} \in R_{\mathrm{O}} w$ for which $M, w^{\prime} \vDash B$. By the induction hypothesis, for all $w^{\prime} \in R_{\mathrm{O}} w, M, w^{\prime} \vDash \neg B$. Hence, by $(\mathrm{CO}), M, w \vDash \mathrm{O} \neg B$. By $(\mathrm{C} \neg \mathrm{P}), M, w \vDash \neg \mathrm{P} B$.

The case $A=\diamond_{J} B$ is analogous and left to the reader.

Theorem 9 (Soundness of PMDL). If $\Gamma \vdash_{\mathbf{P M D L}} A$ then $\Gamma \vdash_{\mathbf{P M D L}} A$.

Proof. Let in the following $M=\left\langle W,\left\langle R_{J}\right\rangle_{J \subseteq \varnothing I}, R_{\mathrm{O}}, v, w\right\rangle$ be a PMDL-model, and $J \subseteq_{\varnothing}$ I. It is sufficient to show that each PMDL-rule holds at the actual world $w$.

$A d(A N D)$ : Suppose $M, w \vDash A, B$, then by $(\mathrm{C} \wedge), M, w \vDash A \wedge B$.

The cases for (AN1), (AN2), (OR1), (OR2), (DM1), (DM2), (DM3), (DM3), $(\mathrm{DN} 1)$, and (DN2) are similar and left to the reader.

Ad (EM): This holds by $(\mathrm{C} \vee)$ and Lemma 1 for all $w^{\prime} \in W$.

$A d\left(4 \square_{J}\right)$ : Suppose $M, w \vDash \square_{J} A$. Hence by $\left(\square_{J}\right)$, for all $w^{\prime} \in R_{J} w, M, w^{\prime} \vDash A$.

Let for some $w^{\prime} \in R_{J} w, R_{J} w^{\prime} w^{\prime \prime}$. Then by the transitivity of $R_{J}$ also $R_{J} w w^{\prime \prime}$ and hence $M, w^{\prime \prime} \vDash A$. Hence, $M, w \vDash \square_{J} \square_{J} A$.

The proof for $\left(4 \diamond_{J}\right)$ is similar and left to the reader.

$A d\left(A N D \square_{J}\right)$ : Suppose $M, w \vDash \square_{J} A$ and $M, w \vDash \square_{J} B$. Hence, by $\left(\mathrm{C} \square_{J}\right)$ and $(\mathrm{C} \wedge)$,

for all $w^{\prime} \in R_{J} w, M, w^{\prime} \vDash A \wedge B$. Hence, again by $\left(\square_{J}\right), M, w \vDash \square_{J}(A \wedge B)$.

The proofs for $\left.\left(\mathrm{AND}^{\prime} \square_{J}\right),\left(\mathrm{OR} \diamond_{J}\right),\left(\mathrm{OR} \square_{J}\right),(\mathrm{ANDO}),(\mathrm{AND})^{\prime}\right),(\mathrm{ORP}),(\mathrm{ORO})$ are analogous and left to the reader.

$A d(D \mathrm{O})$ : Suppose $M, w \vDash \mathrm{O} A$. Hence for all $w^{\prime} \in R_{\mathrm{O}} w, M, w^{\prime} \vDash A$. By the seriality of $R_{0}$ there is such a $w^{\prime} \in R_{0} w$ and hence by $(\mathrm{CP}), M, w \vDash \mathrm{P} A$.

Ad $\left(T \square_{J}\right)$ : Suppose $M, w \vDash \square_{J} A$. By $\left(\square_{J}\right)$ for all $w^{\prime} \in R_{J} w, M, w^{\prime} \vDash A$. Since $R_{J}$ is reflexive, $R_{J} w w$ and hence $M, w \vDash A$.

$A d\left(T \diamond_{J}\right)$ : Suppose $M, w \vDash A$. By the reflexivity of $R_{J}$, also $R_{J} w w$. Hence, by $\left(\mathrm{C} \diamond_{J}\right), M, w \vDash \diamond_{J} A$.

Ad $(R \neg \mathrm{O})$ and $\left(R \mathrm{P}_{\neg}\right)$ : Note that by $\left(\mathrm{C}_{\neg} \mathrm{O}\right), M, w \vDash \neg \mathrm{O} A$ iff $M, w \vDash \mathrm{P}_{\neg} A$.

$A d(R \neg \mathrm{P})$ and $\left(R \mathrm{O}_{\neg}\right)$ : Note that by $(\mathrm{C} \neg \mathrm{P}) M, w \vDash \mathrm{O} \neg A$ iff $M, w \vDash \neg \mathrm{P} A$.

The proofs for $(\mathrm{R} \neg \mathrm{D}),(\mathrm{R} \diamond \neg),(\mathrm{R} \neg \mathrm{P}),(\mathrm{RO} \neg)$ are analogous and left to the reader. 
$A d(R B C)$ : Suppose $A, B \vdash_{\mathbf{P M D L}} D$ and $A, C \vdash_{\mathbf{P M D L}} D$. Suppose further $M, w \vDash A$ and $M, w \vDash B \vee C$. Hence, by $(\mathrm{C} \vee)$ either $M, w \vDash A, B$ or $M, w \vDash A, C$. Hence, by the first supposition, $M, w \vDash D$.

$A d\left(I N H \square_{J}\right)$ : Suppose $A \Vdash \mathbf{P M D L} B$ and $M, w \vDash \square_{J} A$. Hence, by $\left(\mathrm{C} \square_{J}\right)$, for all $w^{\prime} \in R_{J} w, M, w^{\prime} \vDash A$. Let $M^{\prime}=\left\langle W,\left\langle R_{J}\right\rangle_{J \subseteq_{\varnothing} I}, R_{\mathrm{O}}, v, w^{\prime}\right\rangle$. By the definition of $M^{\prime}$, $M^{\prime}, w^{\prime \prime} \vDash C$ iff $M, w^{\prime \prime} \vDash C$ for all $w^{\prime \prime} \in W$. Hence $M^{\prime}, w^{\prime} \vDash A$. Thus, also $M^{\prime} \vDash A$. By the first supposition $M^{\prime} \vDash B$ and hence $M^{\prime}, w^{\prime} \vDash B$. Thus, $M, w^{\prime} \vDash B$. Altogether this shows that $M, w \vDash \square_{J} B$.

The arguments for the rules $\left(\right.$ INH $\left.\diamond_{J}\right),($ INHO) and (INHP) are analogous and left to the reader.

Definition 11. A set $\Gamma$ of formulas is prime iff for all $A \vee B \in \Gamma, \Gamma \cap\{A, B\} \neq \varnothing$.

Where $\mathbf{L}$ is a logic, $\Gamma$ is $\mathbf{L}$-deductively closed iff, if $\Gamma \vdash_{\mathbf{L}} A$ then $A \in \Gamma$.

Definition 12. Let $\Psi_{\text {PMDL }}$ be the set of all prime and PMDL-deductively closed subsets of $\mathcal{L}_{\mathrm{MDL}}$.

Definition 13. We define $\mathbf{R}_{J} \subseteq \Psi_{\mathbf{P M D L}} \times \Psi_{\mathbf{P M D L}}$ as follows: $\mathbf{R}_{J} \Gamma \Delta$ iff (a) whenever $\square_{J} A \in \Gamma$ then $A \in \Delta$, and (b) whenever $A \in \Delta$ then $\diamond_{J} A \in \Gamma$.

Definition 14. We define $\mathbf{R}_{\mathrm{O}} \subseteq \Psi_{\mathbf{P M D L}} \times \Psi_{\mathbf{P M D L}}$ as follows: $\mathbf{R}_{\mathrm{O}} \Gamma \Delta$ iff (a) whenever $\mathrm{O} A \in \Gamma$ then $A \in \Delta$, and (b) whenever $A \in \Delta$ then $\mathrm{P} A \in \Gamma$.

Lemma 2. For all $\Gamma \subseteq \mathcal{L}_{\mathrm{MDL}}, B \in \mathcal{L}_{\mathrm{MDL}}$ we have:

(i) If $\Gamma \vdash \mathbf{P M D L} B$, then $\{\mathrm{O} A \mid A \in \Gamma\} \vdash \mathbf{P M D L} \mathrm{O} B$.

(ii) Where $\Gamma$ is finite, if $\Gamma \vdash_{\mathbf{P M D L}} B$, then $\mathrm{P} \wedge \Gamma \vdash_{\mathbf{P M D L}} \mathrm{P} B$.

(iii) If $\Gamma \vdash \mathbf{P M D L} B$, then $\left\{\square_{J} A \mid A \in \Gamma\right\} \vdash \mathbf{P M D L} \square_{J} B$.

(iv) Where $\Gamma$ is finite, if $\Gamma \vdash_{\mathbf{P M D L}} B$, then $\diamond_{J} \wedge \Gamma \vdash_{\mathbf{P M D L}} \diamond_{J} B$.

Proof. Ad (i): We prove the statement by means of an induction on the number of inference steps $n$ needed to derive $B$ from $\Gamma$ in PMDL.

" $n=1$ ": In case $B$ is derived by a rule $R \notin\left\{(\mathrm{AND}),(\mathrm{ANDO}),\left(\mathrm{AND}^{\prime} \mathrm{O}\right),\left(\mathrm{AND} \square_{J}\right)\right.$, $\left.\left(\mathrm{AND}^{\prime} \square_{J}\right) \mid J \subseteq_{\varnothing} I\right\}$ from some $A \in \Gamma$, then $A \vdash_{\text {PMDL }} B$ and hence by (INHO) also $\mathrm{O} A \vdash_{\mathbf{P M D L}} \mathrm{O} B$.

Suppose $R=(\mathrm{AND})$ and $B$ is derived from $A_{1}, A_{2} \in \Gamma$. Note that $\mathrm{O} A_{1}, \mathrm{O} A_{2}$ $\vdash$ PMDL $\mathrm{O}\left(A_{1} \wedge A_{2}\right)$ by $(\mathrm{ANDO})$.

Suppose $R=(\mathrm{ANDO})$ and $B=\mathrm{O}\left(A_{1} \wedge A_{2}\right)$ is derived from $\mathrm{O} A_{1}, \mathrm{O} A_{2} \in \Gamma$. Then by $(\mathrm{ANDO}), \mathrm{OO} A_{1}, \mathrm{OO} A_{2} \vdash$ PDML $\mathrm{O}\left(\mathrm{O} A_{1} \wedge \mathrm{O} A_{2}\right)$. By (INHO), $\mathrm{O}\left(\mathrm{O} A_{1} \wedge \mathrm{O} A_{2}\right) \vdash$ PMDL $\mathrm{OO}\left(A_{1} \wedge A_{2}\right)$. Altogether, OO $A_{1}, \mathrm{OO} A_{2} \vdash$ PMDL OO $\left(A_{1} \wedge A_{2}\right)$.

Suppose $R=\left(\mathrm{AND}^{\prime} \mathrm{O}\right)$ and $B=\mathrm{P}\left(A_{1} \wedge A_{2}\right)$ is derived from $\mathrm{O} A_{1}, \mathrm{P} A_{2} \in \Gamma$. By (ANDO), OO $A_{1}, \mathrm{OP} A_{2} \vdash \mathrm{PMDL} \mathrm{O}\left(\mathrm{O} A_{1} \wedge \mathrm{P} A_{2}\right)$. By (INHO), $\mathrm{O}\left(\mathrm{O} A_{1} \wedge \mathrm{P} A_{2}\right) \vdash$ PMDL $\mathrm{OP}\left(A_{1} \wedge A_{2}\right)$. Hence, altogether, OO $A_{1}, \mathrm{OP} A_{2} \vdash$ PMDL OP $\left(A_{1} \wedge A_{2}\right)$.

The arguments for $R \in\left\{\left(\mathrm{AND}_{J}\right),\left(\mathrm{AND}^{\prime} \square_{J}\right) \mid J \subseteq_{\varnothing} I\right\}$ are analogous and left to the reader.

" $n \Rightarrow n+1$ ": Suppose $B$ is derived from $\Gamma$ in $n+1$ inference steps from $A_{1}, \ldots, A_{m}$ by means of rule $R$. By the induction hypothesis $\{\mathrm{O} A \mid A \in \Gamma\} \vdash$ PMDL $\mathrm{O} A_{i}$ for all $i \leq m$. If $R \notin\left\{(\mathrm{AND}),(\mathrm{ANDO}),\left(\mathrm{AND}^{\prime} \mathrm{O}\right),\left(\mathrm{AND}_{J}\right),\left(\mathrm{AND}^{\prime} \square_{J}\right) \mid J \subseteq_{\varnothing} I\right\}$, then $m=1$ and $A_{1} \vdash_{\text {PMDL }} B$ and hence by (INHO) also O $A_{1} \vdash_{\text {PMDL }} B$. In case $R \in\{(\mathrm{AND})$, (ANDO), (AND'O), (AND $\left.\left.\square_{J}\right),\left(\mathrm{AND}^{\prime} \square_{J}\right) \mid J \subseteq_{\varnothing} I\right\}$ the argument is analogous to the one given above and is left to the reader.

Ad (ii), (iii) and (iv): This can be shown by an induction similar as in (i) and is left to the reader. 
Definition 15. Where $\Gamma \in \Psi_{\mathrm{PMDL}}$ and $A \in \mathcal{L}_{\mathrm{MDL}}$, let

$$
\begin{aligned}
& \Gamma_{\mathrm{O}}=\{B \mid \mathrm{O} B \in \Gamma\}, \quad \Gamma_{\square}^{J}=\left\{B \mid \square_{J} B \in \Gamma\right\}, \\
& \Gamma_{\mathrm{O}}^{A}=\Gamma_{\mathrm{O}} \cup\{A\} \text {, } \\
& \Gamma_{\mathrm{P}}=\{B \mid \mathrm{P} B \notin \Gamma\} \text {, } \\
& { }^{\vee} \Gamma_{\mathrm{P}}=\left\{\bigvee_{I} B_{i} \mid B_{i} \in \Gamma_{\mathrm{P}}\right\} \text {, } \\
& \Gamma_{\square}^{J, A}=\Gamma_{\square}^{J} \cup\{A\} \text {, } \\
& \Gamma_{\diamond}^{J}=\left\{B \mid \diamond_{J} B \notin \Gamma\right\} \\
& { }^{\vee} \Gamma_{\mathrm{P}}^{A}=\left\{\bigvee_{I} B_{i} \mid B_{i} \in \Gamma_{\mathrm{P}} \cup\{A\}\right\}, \quad{ }^{\vee} \Gamma_{\diamond}^{J, A}=\left\{\bigvee_{I} B_{i} \mid B_{i} \in \Gamma_{\diamond}^{J} \cup\{A\}\right\} .
\end{aligned}
$$

Lemma 3. Let $\Gamma \in \Psi_{\mathrm{PMDL}}$. (i) If $\Gamma_{\mathrm{O}} \vdash_{\mathrm{PMDL}} C$, then $\mathrm{O} C \in \Gamma$. (ii) Where $\mathrm{P} A \in \Gamma$, if $\Gamma_{\mathrm{O}}^{A} \vdash_{\mathrm{PMDL}} C$ then $\mathrm{P} C \in \Gamma$.

Proof. Ad (i): Suppose that $\Gamma_{\mathrm{O}} \vdash_{\mathbf{P M D L}} C$. By Lemma 2i, $\Gamma \vdash_{\mathbf{P M D L}} \mathrm{O} C$. Since $\Gamma$ is PMDL-deductively closed, $\mathrm{O} C \in \Gamma$.

Ad (ii): Suppose $\Gamma_{\mathrm{O}}^{A} \vdash_{\mathbf{P M D L}} C$. Then there is a finite $\Theta \subseteq \Gamma_{\mathrm{O}}$ for which $(\dagger)$ $\Theta \cup\{A\} \vdash$ PMDL $C$. Since $\Theta \subseteq \Gamma_{\mathrm{O}}, \mathrm{O} \wedge \Theta \in \Gamma$ by (ANDO) and the deductive closure of $\Gamma$. Since also $\mathrm{P} A \in \Gamma$, also $\mathrm{P}(\wedge \Theta \wedge A) \in \Gamma$ by $\left(\mathrm{AND}^{\prime} \mathrm{O}\right)$ and the deductive closure of $\Gamma$. Hence, by Lemma 2ii, $(\dagger)$, and the deductive closure of $\Gamma, P C \in \Gamma$.

Lemma 4. Let $\Gamma \in \Psi_{\mathbf{P M D L}}$. (i) If $\Gamma_{\square}^{J} \vdash \mathbf{P M D L} C$, then $\square_{J} C \in \Gamma$. (ii) Where $\diamond_{J} A \in \Gamma$, if $\Gamma_{\square}^{J, A} \vdash \mathbf{P M D L} C$ then $\diamond_{J} C \in \Gamma$.

Proof. Analogous to Lemma 3. We use $\left(\mathrm{AND}_{J}\right),\left(\mathrm{AND}^{\prime} \square_{J}\right)$, Lemma 2iii, and Lemma 2iv instead of (ANDO), (AND'O), Lemma 2i, and Lemma 2ii respectively.

Lemma 5. Let $\Gamma \in \Psi_{\mathrm{PMDL}}$. (i) Where $\mathrm{P} A \in \Gamma,{ }^{\vee} \Gamma_{\mathrm{P}} \cap \mathrm{Cn}_{\mathrm{PMDL}}\left(\Gamma_{\mathrm{O}}^{A}\right)=\varnothing$. (ii) Where $B \notin \Gamma_{\mathrm{O}},{ }^{\vee} \Gamma_{\mathrm{P}}^{B} \cap \mathrm{Cn}_{\mathrm{PMDL}}\left(\Gamma_{\mathrm{O}}\right)=\varnothing$.

Proof. Ad (i): Let $C=\bigvee_{I} C_{i}$ where $C_{i} \in \Gamma_{\mathrm{P}}$ for all $i \in I$. Assume $C \in \operatorname{Cn}_{\mathrm{PMDL}}\left(\Gamma_{\mathrm{O}}^{A}\right)$ then by Lemma 3ii, $\mathrm{P} \vee{ }_{I} C_{i} \in \Gamma$. Hence, by (ORP) and the deductive closure of $\Gamma$, also $\vee_{I} \mathrm{P} C_{i} \in \Gamma$. Since $\Gamma$ is prime, there is an $i \in I$ such that $\mathrm{P} C_{i} \in \Gamma$ and hence $C_{i} \notin \Gamma_{\mathrm{P}},-\mathrm{a}$ contradiction.

Ad (ii): Let $C=\bigvee_{I} C_{i}$ where $C_{i} \in \Gamma_{\mathrm{P}} \cup\{B\}$ for all $i \in I$. Assume $C \in \mathrm{Cn}_{\mathrm{PMDL}}\left(\Gamma_{\mathrm{O}}\right)$. By Lemma $3 \mathrm{i}$ and the deductive closure of $\Gamma,(\star) \mathrm{O} \vee_{I} C_{i} \in \Gamma$. Assume that all $C_{i} \in \Gamma_{\mathrm{P}}$. By (DO), $\mathrm{P} \vee_{I} C_{i} \in \Gamma$. By (ORP), $\vee_{I} \mathrm{P} C_{i} \in \Gamma$. Since $\Gamma$ is prime there is a $i \in I$ for which $\mathrm{P}_{i} \in \Gamma$ and hence $C_{i} \notin \Gamma_{\mathrm{P}},-$ a contradiction. Thus, there is a non-empty $J \subseteq I$ such that for each $j \in J, C_{j}=B$, and for each $j \in I \backslash J, C_{j} \neq B$. By $(\star)$, (ORO) and the deductive closure of $\Gamma, \mathrm{O} B \vee \mathrm{P} \vee{ }_{I \backslash J} C_{i} \in \Gamma$. Hence, by (ORP), $\mathrm{O} B \vee \vee_{I \backslash J} \mathrm{P} C_{i} \in \Gamma$. Since $B \notin \Gamma$ ond since $\Gamma$ is prime, there is an $i \in I \backslash J$ such that $\mathrm{P} C_{i} \in \Gamma$ and hence $C_{i} \notin \Gamma_{\mathrm{P}},-$ a contradiction.

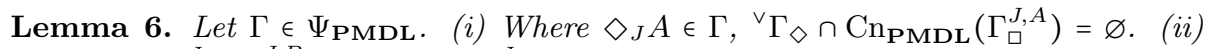
Where $B \notin \Gamma_{\square}^{J},{ }^{\vee} \Gamma_{\diamond}^{J, B} \cap \mathrm{Cn}_{\mathbf{P M D L}}\left(\Gamma_{\square}^{J}\right)=\varnothing$.

Proof. The proof is analogous to the proof of Lemma 5. We use Lemma 4, (OR $\left.\diamond_{J}\right)$, $\left(\mathrm{D} \square_{J}\right)$, and $\left(\mathrm{OR} \square_{J}\right)$ instead of Lemma 3, (ORP), (DO), and (ORO) respectively.

Lemma 7. Let $\Gamma \in \Psi_{\mathbf{P M D L}}$.

(i) Where $\mathrm{P} A \in \Gamma$, there is $a \Delta \subseteq \mathcal{L}_{\mathrm{MDL}}$ for which (1) $\Gamma_{\mathrm{O}}^{A} \subseteq \Delta$, (2) ${ }^{\vee} \Gamma_{\mathrm{P}} \cap \Delta=\varnothing$, and (3) $\Delta \in \Psi_{\mathbf{P M D L}}$.

(ii) Where $B \notin \Gamma_{\mathrm{O}}$, there is a $\Delta \subseteq \mathcal{L}_{\mathrm{MDL}}$ for which (1) $\Gamma_{\mathrm{O}} \subseteq \Delta$, (2) ${ }^{\vee} \Gamma_{\mathrm{P}}^{B} \cap \Delta=\varnothing$, and (3) $\Delta \in \Psi_{\text {PMDL }}$. 
Proof. Let $\left\langle\boldsymbol{\Gamma}_{\mathbf{O}}, \boldsymbol{\Gamma}_{\mathbf{P}}\right\rangle \in\left\{\left\langle\Gamma_{\mathrm{O}}^{A},{ }^{\vee} \Gamma_{\mathrm{P}}\right\rangle,\left\langle\Gamma_{\mathrm{O}},{ }^{\vee} \Gamma_{\mathrm{P}}^{B}\right\rangle\right\}$. Where $\left\langle B_{1}, B_{2}, \ldots\right\rangle$ is a list of all the members of $\mathcal{L}_{\mathrm{MDL}}$, define $\Delta_{0}=\mathrm{Cn}_{\mathrm{PMDL}}\left(\boldsymbol{\Gamma}_{\mathbf{O}}\right)$ and $\Delta=\Delta_{0} \cup \Delta_{1} \cup \ldots$ where

$$
\Delta_{i+1}= \begin{cases}\operatorname{Cn}_{\mathbf{P M D L}}\left(\Delta_{i} \cup\left\{B_{i+1}\right\}\right) & \text { if } \boldsymbol{\Gamma}_{\mathbf{P}} \cap \operatorname{Cn}_{\mathbf{P M D L}}\left(\Delta_{i} \cup\left\{B_{i+1}\right\}\right)=\varnothing \\ \Delta_{i} & \text { else }\end{cases}
$$

Ad (1): This holds by the definition of $\Delta_{0}$ and since $\Delta_{0} \subseteq \Delta$.

Ad (2): By Lemma $5, \Delta_{0} \cap \boldsymbol{\Gamma}_{\mathbf{P}}=\varnothing$. The rest follows by the construction of $\Delta$.

Ad (3): We first show that $\Delta$ is PMDL-deductively closed. Assume there is a $B_{i} \notin \Delta$ for which $(\dagger) \Delta \vdash_{\mathbf{P M D L}} B_{i}$. Then, by the construction, there is a $D \in \boldsymbol{\Gamma}_{\mathbf{P}}$ for which $\Delta \cup\left\{B_{i}\right\} \vdash_{\text {PMDL }} D$. Hence, by $(\dagger), \Delta \vdash_{\text {PMDL }} D$. Hence, there is a $j \in \mathbb{N}$ such that $\Delta_{j} \vdash_{\mathbf{P M D L}} D$. By the construction $\Delta_{j}=\mathrm{Cn}_{\mathbf{P M D L}}\left(\Delta_{j}\right)$ and thus, $D \in \Delta_{j}$. Hence, $D \in \Delta,-$ a contradiction with (2).

We now show that $\Delta$ is prime. Suppose $A_{1} \vee A_{2} \in \Delta$. Assume that $A_{1}, A_{2} \notin \Delta$. By the construction, $\Delta \cup\left\{A_{1}\right\} \vdash$ PMDL $D_{1}$ and $\Delta \cup\left\{A_{2}\right\} \vdash$ PMDL $D_{2}$ for some $D_{1}, D_{2} \in \Gamma_{\mathbf{P}}$. Hence, by (OR1), $\Delta \cup\left\{A_{1}\right\} \vdash$ PMDL $D_{1} \vee D_{2}$ and by (OR2) $\Delta \cup\left\{A_{2}\right\} \vdash$ PMDL $D_{1} \vee D_{2}$. Hence, by Fact $1, \Delta \cup\left\{A_{1} \vee A_{2}\right\} \vdash$ PMDL $D_{1} \vee D_{2}$ and since $A_{1} \vee A_{2} \in \Delta, \Delta \vdash$ PMDL $D_{1} \vee D_{2}$ and hence $D_{1} \vee D_{2} \in \Delta$ by the deductive closure of $\Delta$. However, $D_{1} \vee D_{2} \in \boldsymbol{\Gamma}_{\mathbf{P}}$, a contradiction with (2).

Lemma 8. Let $\Gamma \in \Psi_{\mathrm{PMDL}}$.

(i) Where ${ }_{J} A \in \Gamma$, there is a $\Delta \subseteq \mathcal{L}_{\mathrm{MDL}}$ for which (1) $\Gamma_{\square}^{J, A} \subseteq \Delta$, (2) ${ }^{\vee} \Gamma_{\diamond}^{J} \cap \Delta=\varnothing$, and (3) $\Delta \in \Psi_{\text {PMDL }}$.

(ii) Where $B \notin \Gamma_{\square}^{J}$, there is a $\Delta \subseteq \mathcal{L}_{\mathrm{MDL}}$ for which (1) $\Gamma_{\square}^{J} \subseteq \Delta$, (2) ${ }^{\vee} \Gamma_{\diamond}^{J, B} \cap \Delta=\varnothing$, and (3) $\Delta \in \Psi_{\text {PMDL. }}$.

Proof. The proof is analogous to the proof of Lemma 7. Instead of making use of Lemma 5 we now use Lemma 6.

Lemma 9. Where $\Gamma \in \Psi_{\mathbf{P M D L}}, \mathrm{P} A \in \Gamma$ iff there is a $\Delta \in \Psi_{\mathbf{P M D L}}$ such that $\mathbf{R}_{\mathrm{O}} \Gamma \Delta$ and $A \in \Delta$.

Proof. Left-Right: Suppose $\mathrm{P} A \in \Gamma$. By Lemma 7i there is a $\Delta \subseteq \mathcal{L}_{\mathrm{MDL}}$ for which (1) $\Gamma_{\mathrm{O}}^{A} \subseteq \Delta$, (2) for all $C \in \Gamma_{\mathrm{P}}, C \notin \Delta$, and (3) $\Delta \in \Psi_{\mathrm{PMDL}}$. We now show that $\mathbf{R}_{\mathrm{O}} \Gamma \Delta$. Ad (a): if, for some $D, \mathrm{O} D \in \Gamma$, then $D \in \Gamma_{\mathrm{O}}^{A}$ and hence $D \in \Delta$ by (1). Ad (b): suppose $\mathrm{P} E \notin \Gamma$ for some $E \in \mathcal{L}_{\mathrm{MDL}}$. Then $E \in \Gamma_{\mathrm{P}}$ and thus $E \notin \Delta$ by (2).

Right-Left: follows directly by the definition of $\mathbf{R}_{O}$.

Lemma 10. Where $\Gamma \in \Psi_{\mathbf{P M D L}}, \diamond_{J} A \in \Gamma$ iff there is a $\Delta \in \Psi_{\mathbf{P M D L}}$ such that $\mathbf{R}_{J} \Gamma \Delta$ and $A \in \Delta$.

Proof. The proof is analogous to the proof of Lemma 9, except that we use Lemma $8 \mathrm{i}$ instead of Lemma $7 \mathrm{i}$.

Lemma 11. For every $\Gamma \in \Psi_{\mathbf{P M D L}}$ there is a $\Delta \in \Psi_{\mathbf{P M D L}}$ such that $\mathbf{R}_{\bigcirc} \Gamma \Delta .\left(\mathbf{R}_{\bigcirc}\right.$ is serial.)

Proof. By $(\mathrm{EM})$ and (INHP), $\vdash$ PDML $\mathrm{P}(A \vee \neg A)$. Hence, $\mathrm{P}(A \vee \neg A) \in \Gamma$ by the deductive closure of $\Gamma$. By Lemma 9 , there is a $\Delta \in \Psi_{\mathbf{P M D L}}$ such that $\mathbf{R}_{\bigcirc} \Gamma \Delta$ and $A \vee \neg A \in \Delta$.

Lemma 12. Where $\Gamma \in \Psi_{\mathbf{P M D L}}, \mathrm{O} A \in \Gamma$ iff, for all $\Delta \in \Psi_{\mathrm{PMDL}}$ such that $\mathbf{R}_{\mathrm{O}} \Gamma \Delta$, $A \in \Delta$. 
Proof. Left-Right: This is an immediate consequence of the definition of $\mathbf{R}_{0}$.

Right-Left: Suppose $\mathrm{O} A \notin \Gamma$. Hence $A \notin \Gamma_{\mathrm{O}}$. By Lemma 7ii, there is a $\Delta \subseteq \mathcal{L}_{\mathrm{MDL}}$ for which (1) $\Gamma_{\mathrm{O}} \subseteq \Delta,(2)\left(\Gamma_{\mathrm{P}} \cup\{A\}\right) \cap \Delta=\varnothing$, and (3) $\Delta \in \Psi_{\text {PMDL }}$. We now show that $\mathbf{R}_{\mathrm{O}} \Gamma \Delta$. Ad (a): if, for some $D \in \mathcal{L}_{\mathrm{MDL}}, \mathrm{O} D \in \Gamma$, then $D \in \Gamma_{\mathrm{O}}$ and thus $D \in \Delta$ by (1). Ad (b): Suppose $\mathrm{P} E \notin \Gamma$ and hence $E \in \Gamma_{\mathrm{P}}$. Thus, $E \notin \Delta$ by (2).

Lemma 13. Where $\Gamma \in \Psi_{\mathbf{P M D L}}, \square_{J} A \in \Gamma$ iff, for all $\Delta \in \Psi_{\mathbf{P M D L}}$ such that $\mathbf{R}_{J} \Gamma \Delta$, $A \in \Delta$.

Proof. The proof is analogous to the proof of Lemma 12, except that instead of Lemma 7ii we make use of Lemma 8ii.

Lemma 14. Where $\Gamma \in \Psi_{\mathbf{P M D L}}, \mathbf{R}_{J} \Gamma \Gamma$. ( $\mathbf{R}_{J}$ is reflexive.)

Proof. Assume there is a $\Gamma \in \Psi_{\mathbf{P M D L}}$ for which $\mathbf{R}_{J} \Gamma \Gamma$ is not the case. Then, either (1) there is a $\square_{J} A \in \Gamma$ such that $A \notin \Gamma$, or (2) there is a $A \in \Gamma$ such that $\diamond_{J} A \notin \Gamma$. Ad

(1): Since $\Gamma$ is PMDL-deductively closed, and by $\left(\mathrm{T}_{J}\right), \square_{J} A \vdash A$, also $A \in \Gamma$. Ad (2): Since $\Gamma$ is PMDL-deductively closed, and by $\left(\mathrm{T} \diamond_{J}\right), A \vdash \diamond_{J} A$, also $\diamond_{J} A \in \Gamma$. Since neither (1) nor (2) we reached a contradiction.

Lemma 15. If $\mathbf{R}_{J} \Gamma \Delta$ and $\mathbf{R}_{J} \Delta \Delta^{\prime}$ then $\mathbf{R}_{J} \Gamma \Delta^{\prime}$. ( $\mathbf{R}_{J}$ is transitive.)

Proof. Suppose $\mathbf{R}_{J} \Gamma \Delta$ and $\mathbf{R}_{J} \Delta \Delta^{\prime}$. Assume not $\mathbf{R}_{J} \Gamma \Delta^{\prime}$. Thus, either (1) there is a $\square_{J} A \in \Gamma$ for which $A \notin \Delta^{\prime}$, or (2) there is a $A \in \Delta^{\prime}$ for which $\diamond_{J} A \notin \Gamma$. Ad (1): Suppose $\square_{J} A \in \Gamma$. By $\left(4 \square_{J}\right)$ and the PMDL-deductive closure of $\Gamma$, also $\square_{J} \square_{J} A \in \Gamma$. Hence, by (a) in the definition of $\mathbf{R}_{J}, \square_{J} A \in \Delta$. Hence, again by (a) in the definition of $\mathbf{R}_{J}$ and since $\mathbf{R}_{J} \Delta \Delta^{\prime}, A \in \Delta^{\prime}$. Ad (2): Suppose $A \in \Delta^{\prime}$. Hence $\diamond_{J} A \in \Delta$ by (b) in the definition of $\mathbf{R}_{J}$ and since $\mathbf{R}_{J} \Delta \Delta^{\prime}$. Hence, $\diamond_{J} \diamond_{J} A \in \Gamma$ by (b) in the definition of $\mathbf{R}_{J}$ and since $\mathbf{R}_{J} \Gamma \Delta$. Since $\left(4 \diamond_{J}\right)$ is valid in $\Gamma$, also $\diamond_{J} A \in \Gamma$.

Since neither (1) nor (2) is the case we reached a contradiction.

Lemma 16. Where $\Delta \in \Psi_{\mathbf{P M D L}}$, there is a PMDL-model $M$ such that $M \vDash A$ for all $A \in \Delta$ and $M \not A$ for all $A \in \mathcal{L}_{\mathrm{MDL}} \backslash \Delta$.

Proof. Let $\Delta \in \Psi_{\mathbf{P M D L}}$. We construct a PMDL-model

$$
M=\left\langle\Psi_{\mathbf{P M D L}},\left\langle\mathbf{R}_{J}\right\rangle_{J \subseteq_{\varnothing} I}, \mathbf{R}_{\mathrm{O}}, v, \Delta\right\rangle
$$

such that ( $\dagger$ ) for all $A \in \mathcal{P}^{\urcorner}, w \in v(A)$ iff $A \in w$. By Lemmas 11, 14, 15, $\mathbf{R}_{\mathrm{O}}$ and $\mathbf{R}_{J}$ (for all $J \subseteq_{\varnothing} I$ ) have the needed properties for $M$ to be a PMDL-model.

We now show by an induction that for all $w \in \Psi_{\text {PMDL }}$ and for all $A \in \mathcal{L}_{\text {MDL }}$, $M, w \vDash A$ iff $A \in w$. The induction is in terms of the length of the formulas $A \in \mathcal{L}_{\mathrm{MDL}}$ in question.

Let $A \in \mathcal{P}$. By $(\dagger), A \in w$ iff $w \in v(A)$ iff [by $(\mathrm{C} \mathcal{P})] M, w \vDash A$.

For the induction step let first $A$ be of the form $\neg B$.

Let first $B \in \mathcal{P}$. By $(\dagger), \neg B \in w$ iff $w \in v(\neg B)$. By $\left(\mathrm{C}^{\prime}\right)$, if $\neg B \in w$ and hence $w \in v(\neg B)$, then $M, w \vDash \neg B$. Suppose now that $M, w \vDash \neg B$. By $\left(\mathrm{C}_{\neg}^{\prime}\right)$ either $w \in v(\neg B)$ and hence $\neg B \in w$, or $M, w \notin B$. In the second case, by the induction hypothesis, $B \notin w$. Since $w$ is prime and since $B \vee \neg B \in w$ (since $w$ is PMDL-deductively closed), $\neg B \in w$.

Now let $B=\neg B^{\prime} . \quad M, w \vDash \neg \neg B^{\prime}$ iff [by $(\mathrm{C} \neg \neg)$ ] $M, w \vDash B^{\prime}$ iff [by the induction hypothesis] $B^{\prime} \in w$ iff [since $w$ is DPML-deductively closed, (DN1) and (DN2)] $\neg \neg B^{\prime} \in$ $w$.

The cases $B \in\left\{B_{1} \wedge B_{2}, B_{1} \vee B_{2}\right\}$ are similar and left to the reader. 
Let now $B=\mathrm{O} B^{\prime} . M, w \vDash \neg \mathrm{O} B^{\prime}$ iff [by $\left.\left(\mathrm{C}_{\neg} \mathrm{O}\right)\right] M, w \vDash \mathrm{P}_{\neg} B^{\prime}$ iff [by $(\mathrm{CP})$ ] there is a $w^{\prime} \in \mathbf{R}_{\mathrm{O}} w$ for which $M, w^{\prime} \vDash \neg B^{\prime}$ iff [by the induction hypothesis] $\neg B^{\prime} \in w^{\prime}$ iff [by the definition of $\mathbf{R}_{0}$ ] $\mathrm{P}_{\neg} B^{\prime} \in w$ iff [by $(\mathrm{R} \neg \mathrm{O})$ and $\left(\mathrm{RP}_{\neg}\right)$ ] $\neg \mathrm{O} B^{\prime} \in w$.

The case $B=\square_{J} B^{\prime}$ is analogous.

Let now $B=\mathrm{P} B^{\prime} . \quad M, w \vDash \neg \mathrm{P} B^{\prime}$ iff [by $\left.(\mathrm{C} \neg \mathrm{P})\right] M, w \vDash \mathrm{O}_{\neg} B^{\prime}$ iff [by $(\mathrm{CO})$ ] for all $w^{\prime} \in \mathbf{R}_{\circ} w, M, w^{\prime} \vDash \neg B^{\prime}$ iff [by the induction hypothesis] for all $w^{\prime} \in \mathbf{R}_{\mathrm{O}} w, \neg B^{\prime} \in w^{\prime}$ iff [by Lemma 12] $\mathrm{O} \neg B^{\prime} \in w$ iff $[$ by $(\mathrm{R} \neg \mathrm{P})$ and $(\mathrm{RO} \neg)] \neg \mathrm{P} B^{\prime} \in w$.

The case $B=\diamond_{J} B^{\prime}$ is analogous (except that we use Lemma 13 instead of Lemma 12).

Let now $A=B \wedge C . \quad M, w \vDash B \wedge C$ iff [by $(\mathrm{C} \wedge)$ ] $M, w \vDash B$ and $M, w \vDash C$ iff [by the induction hypothesis] $B, C \in w$ iff [by (AND), (AN1), (AN2) and the fact that $w$ is PMDL-deductively closed] $B \wedge C \in w$.

The case $A=B \vee C$ is similar and left to the reader.

Let $A=\mathrm{O} B$. $M, w \vDash \mathrm{O} B$ iff [by $(\mathrm{CO})$ ] for all $w^{\prime} \in \mathbf{R}_{\mathrm{O}} w, M, w^{\prime} \vDash B$ iff [by the induction hypothesis] for all $w^{\prime} \in \mathbf{R}_{\mathrm{O}} w, B \in w^{\prime}$ iff [by Lemma 12] $\mathrm{O} B \in w$.

The case $A=\square_{J} B$ is analogous and left to the reader (just we use Lemma 13 instead of Lemma 12).

Let $A=\mathrm{P} B . \quad M, w \vDash \mathrm{P} B$ iff $\left[\right.$ by $(\mathrm{CP})$ ] there is a $w^{\prime} \in \mathbf{R}_{\mathrm{O}} w$ for which $M, w^{\prime} \vDash B$ iff [by the induction hypothesis] $B \in w^{\prime}$ iff [by Lemma 9] $\mathrm{P} B \in w$.

The case $A=\diamond_{J} B$ is analogous (just we use Lemma 10 instead of Lemma 9).

Lemma 17. Let $\Gamma \subseteq \mathcal{L}_{\mathrm{MDL}}$ and $\Gamma \nvdash_{\mathrm{PMDL}} A$. There is a $\Delta \subseteq \mathcal{L}_{\mathrm{MDL}}$ such that (i) $\Gamma \subseteq \Delta$, (ii) $A \notin \Delta$, and (iii) $\Delta \in \Psi_{\mathrm{PMDL}}$.

Proof. Where $\left\langle B_{1}, B_{2}, \ldots\right\rangle$ is a list of the members of $\mathcal{L}_{\mathrm{MDL}}$, define $\Delta_{0}=\operatorname{Cn}_{\mathbf{P M D L}}(\Gamma)$ and $\Delta=\Delta_{0} \cup \Delta_{1} \cup \ldots$, where

$$
\Delta_{i+1}= \begin{cases}\operatorname{Cn}_{\mathbf{P M D L}}\left(\Delta_{i} \cup\left\{B_{i+1}\right\}\right) & \text { if } A \notin \operatorname{Cn}_{\mathbf{P M D L}}\left(\Delta_{i} \cup\left\{B_{i+1}\right\}\right) \\ \Delta_{i} & \text { else }\end{cases}
$$

Ad (i): This holds by the definition of $\Delta_{0}$ and since $\Delta_{0} \subseteq \Delta$.

Ad (ii): This holds since $A \notin \mathrm{Cn}_{\mathrm{PMDL}}(\Gamma)$ and by the construction of $\Delta$.

Ad (iii): Assume that some $B_{i} \notin \Delta$ but $\Delta \vdash$ PMDL $B_{i}$. Hence, by the construction of $\Delta, \Delta_{i-1} \cup\left\{B_{i}\right\} \vdash$ PMDL $A$ and hence $\Delta \cup\left\{B_{i}\right\} \vdash$ PMDL $A$. Since also $\Delta \vdash$ PMDL $B_{i}$, $\Delta \vdash$ PDML $A,-$ a contradiction with (ii). Hence $\Delta$ is PMDL-deductively closed.

Suppose $B \vee C \in \Delta$. Assume $B, C \notin \Delta$. Hence, $\Delta \cup\{B\} \vdash$ PMdL $A$ and $\Delta \cup$ $\{C\} \vdash$ PMDL $A$. Hence, by Fact $1, \Delta \cup\{B \vee C\} \vdash$ PMDL $A$ and since $B \vee C \in \Delta$ also $\Delta \vdash$ PMDL $A,-$ a contradiction with (ii). Hence, $\Delta$ is prime.

Theorem 10 (Strong Completeness of PMDL.). If $\Gamma \Vdash \mathbf{P M D L} A$ then $\Gamma \vdash_{\mathbf{P M D L}} A$.

Proof. Suppose $\Gamma H_{\text {PMDL }} A$. By Lemma 17 there is a $\Delta \supseteq \Gamma$ such that $A \notin \Delta$ and $\Delta \in \Psi_{\mathbf{P M D L}}$. By Lemma 16, there is a PMDL-model $M$ for which $M \vDash B$ for all $B \in \Delta$ and $M \not A$.

\section{Proof of Theorem 8}

The following fact holds since MDL strengthens classical propositional logic.

Fact 2. $\Gamma \vdash \mathrm{MDL} \neg A \vee B$ iff $\Gamma \cup\{A\} \vdash \mathrm{MDL} B$.

Lemma 18. The following is valid in ULL: 
(i) $A, A \supset B \vdash \mathbf{U L L} B$

(ii) If $A \vdash \mathbf{U L L} B$ then $\vdash$ ULL $A \supset B$.

(iii) If $\vdash$ ULL $A \supset B$ then $A \vdash \mathbf{u L L} B$.

(iv) If $A \vdash \mathbf{U L L} B$ then $\neg B \vdash \mathbf{U L L} \neg A$.

Proof. Ad (i): Suppose $A$ and $\neg A \vee B$. (1) Suppose $\neg A$. By $A$ and $\neg A$ we get $B$ by means of (ECQ). (2) Suppose now $B$, then by (AND) and (AN1), B. By (1), (2), (RBC), and the supposition, B. Ad (ii): Suppose $A \vdash_{\text {UlL }} B$. (1) Hence, by (OR2) and the supposition, $A \vdash \mathrm{ULL} \neg A \vee B$. (2) By (OR1) $\neg A \vdash \mathrm{ULL} \neg A \vee B$. (3) By (EM), $A \vee \neg A$. By (1), (2), (3) and (RBC), $\vdash \mathbf{U L L} \neg A \vee B$. Ad (iii): Suppose $\vdash$ ULL $\neg A \vee B$. Suppose $A$. By (i), B. Hence $A \vdash$ ull $B$. Ad (iv): Suppose $A \vdash$ ull B. By (ii), $\vdash \mathbf{U L L} \neg A \vee B$. By (RBC) and (DN1), $\vdash \mathbf{U L L} \neg A \vee \neg \neg B$. By (OR1), (OR2), and (RBC), $\vdash \mathrm{ULL} \neg \neg B \vee \neg A$. By (iii), $\neg B \vdash \mathrm{ULL} \neg A$.

Proof of Theorem 8. We first show that all the MDL axioms are valid in ULL.

By Lemma 18.i and the fact that all classical theorems are theorems of LP (see e.g., [33]), ULL strengthens CL. Let in the following $\square \in\left\{O, \square_{J} \mid J \subseteq_{\varnothing} I\right\}$ and $\diamond \in\left\{\mathrm{P}, \diamond_{J} \mid J \subseteq_{\varnothing} I\right\}$.

$A d(A K \bullet):$ By simple propositional manipulations (henceforth, SPM), $(\neg A \vee$ $B) \vdash \mathrm{ULL} \odot(B \vee \neg A)$. By (OR๑), $\odot(\neg A \vee B) \vdash \mathrm{ULL} \odot B \vee \odot \neg A$. By $(\mathrm{R} \odot \neg)$ and some SPM, $\odot(\neg A \vee B) \vdash \mathbf{U L L} \neg \odot A \vee \boxminus B$. By Lemma 18.ii, $\vdash \mathbf{U L L} \odot(\neg A \vee B) \supset(\neg \odot A \vee \boxminus B) . A d$ $\left(A 4 \square_{J}\right)$ : This follows by Lemma 18.ii and $\left(4 \square_{J}\right)$. Ad $\left(A \square_{J}\right)$ : This follows by Lemma 18.ii and $\left(\mathrm{AT} \square_{J}\right) . A d(A D f \odot):$ By $(\mathrm{R} \odot \neg)$ and Lemma 18.iv, $\neg \neg \odot A \vdash \mathrm{ULL} \neg ๑ \neg A$. By $(\mathrm{DN} 1), \diamond A \vdash \mathbf{U L L} \neg \neg \odot A$. Hence, $\odot A \vdash \mathbf{U L L} \neg \boxminus \neg A$. By Lemma 18.ii, $\vdash$ ULL $\odot A \supset$ $\neg \odot \neg A$. In a similar way we get $\vdash$ ULL $\neg \odot \neg A \supset \odot A$. By (AND), $\vdash$ ULL $\odot A \equiv \neg \odot \neg A$. $A d(N E C \bullet)$ : This follows by (INH๑). Ad (ADO): This follows by (DO) and Lemma 18.ii.

We now show that all the ULL axioms are valid in MDL.

All the rules and axioms of LP hold trivially in MDL due to the fact that MDL strengthens CL.

$A d\left(4 \square_{J}\right),\left(4 \diamond_{J}\right),\left(T \square_{J}\right),\left(T \diamond_{J}\right),(D \mathrm{O})$ : This follows by Fact 2 and $\left(\mathrm{A} 4 \square_{J}\right)$, $\left(\mathrm{A} 4 \diamond_{J}\right),\left(\mathrm{AT} \square_{J}\right)$, and (ADO). Ad (INH๑): This follows by (NEC $\left.\bullet\right),(\mathrm{AK} \bullet)$ and SPM. Ad $(\mathrm{INH} \odot)$ : This follows by (INH๑), (ADf $\odot)$ and SPM. Ad (AND๑): This follows by (NEC $\bullet),(\mathrm{AK} \bullet)$ and by Fact $2 . A d\left(A N D^{\prime} \bullet\right)$ : By (ADfP), (AND๑), by Fact 2 and $\mathrm{SPM}, \varpi A, \neg \odot(A \wedge B) \vdash \mathrm{MDL} \odot(A \wedge \neg B)$. By $(\mathrm{INH}), \odot(A \wedge \neg B) \vdash \mathrm{MDL} \bullet \neg B$. By $(\mathrm{ADf} \odot)$ and SPM, $\odot(A \wedge \neg B) \vdash \mathrm{MDL} \neg \odot B$. Altogether, $\bullet A, \neg \diamond(A \wedge B) \vdash_{\mathrm{MDL}} \neg \diamond B$. By SPM, $\odot A, \odot B \vdash$ MDL $\odot(A \wedge B)$. Ad $(R \neg \odot),(R \odot \neg),(R \bullet \neg),(R \neg \odot)$ : This follows by $(\mathrm{ADf} \odot)$ ) and SPM. $A d(\mathrm{OR} \odot)$ : By (AND๑), $\neg A \wedge \bullet \neg B \vdash \mathrm{MDL} \bullet(\neg A \wedge \neg B)$. By Contraposition, (ADf $\odot)$, and SPM, $\odot(A \vee B) \vdash \mathrm{MDL} \odot A \vee \odot B . A d(O R \odot):$ By SPM, $\odot(A \vee B) \vdash \mathrm{MDL} \odot(\neg B \supset A)$. By (AK๑) and by Fact $2, \odot(\neg B \supset A) \vdash \mathrm{MDL} \neg \bullet \neg B \vee \odot A$. By SPM and (ADf $\odot), \neg \odot \neg B \vee \odot A \vdash$ MDL $\odot A \vee \diamond B$. Altogether, $\odot(A \vee B) \vdash$ MDL $\odot A \vee \odot B$.

\section{References}

[1] P. Bailhache. Authorities and addressees in deontic logic: indexed operators and action. In J.-J. Ch. Meyer and R.J. Wieringa, editors, Proceedings of the First International Workshop on Deontic Logic in Computer Science, pages $72-$ 88, 1991. 
[2] Diderik Batens. A survey of inconsistency-adaptive logics. In Diderik Batens, Graham Priest, and Jean-Paul van Bendegem, editors, Frontiers of Paraconsistent Logic, pages 49-73. Baldock: Research Studies Press, Kings College Publication, 2000.

[3] Diderik Batens. A universal logic approach to adaptive logics. Logica Universalis, 1:221-242, 2007.

[4] Diderik Batens. Towards a dialogic interpretation of dynamic proofs. In Cédric Dégremont, Laurent Keiff, and Helge Rückert, editors, Dialogues, Logics and Other Strange Things. Essays in Honour of Shahid Rahman, pages 27-51. College Publications, London, 2009.

[5] Diderik Batens and Joke Meheus. Recent results by the inconsistency-adaptive labourers. In Jean-Yves Béziau, Walter Carnielli, and Dov Gabbay, editors, Handbook of Paraconsistency, pages 81-99. College Publications, London, 2007.

[6] Mathieu Beirlaen and Christian Straßer. A paraconsistent multi-agent framework for dealing with normative conflicts. In João Leite, Paolo Torroni, Thomas Ågotnes, Guido Boella, and Leon van der Torre, editors, Computational Logic in Multi-Agent Systems. Proceedings of the $12^{\text {th }}$ International Workshop CLIMA XII, volume 6814 of Lecture Notes in Artificial Intelligence, pages 312-329. Springer-Verlag, 2011.

[7] Mathieu Beirlaen, Christian Straßer, and Joke Meheus. An inconsistency-adaptive deontic logic for normative conflicts. Journal of Philosophical Logic. In print. DOI: 10.1007/s10992-011-9221-3.

[8] Nuel Belnap and Michael Perloff. In the realm of agents. Annals of Mathematics and Artificial Intelligence, 9:25-48, 1993.

[9] Nuel Belnap, Michael Perloff, and Ming Xu. Facing the Future. Agents and Choices in Our Indeterminist World. Oxford University Press, 2001.

[10] Jan Broersen. Action negation and alternative reductions for dynamic deontic logics. Journal of Applied Logic, 2:153-168, 2004.

[11] Newton Da Costa and Walter Carnielli. On paraconsistent deontic logic. Philosophia, 16:293-305, 1986.

[12] Frank Dignum and Lambèr Royakkers. Collective commitment and obligation. In C. Ciampi and E. Marinai, editors, Proceedings of 5th Int. conference on Law in the Information Society, Firenze, Italy, pages 1008-1021, 1998.

[13] Bas C. Van Fraassen. Values and the heart's command. Journal of Philosophy, 70:5-19, 1973.

[14] Lou Goble. A proposal for dealing with deontic dilemmas. In Alessio Lomuscio and Donald Nute, editors, DEON, volume 3065 of Lecture Notes in Computer Science, pages 74-113. Springer, 2004.

[15] Lou Goble. A logic for deontic dilemmas. Journal of Applied Logic, 3:461-483, 2005.

[16] Lou Goble. Normative conflicts and the logic of ought. Nô̂s, 43:450-489, 2009.

[17] Christopher W. Gowans, editor. Moral Dilemmas. Oxford University Press, 1987.

[18] Jörg Hansen. Conflicting imperatives and dyadic deontic logic. Journal of Applied Logic, 3:484-511, 2005. 
[19] Bengt Hansson. Deontic logic and different levels of generality. Theoria, 36(3):241-248, 1970.

[20] Sven Ove Hansson. The Structure of Values and Norms. Cambridge University Press, 2001.

[21] John F. Horty. Moral dilemmas and nonmonotonic logic. Journal of Philosophical Logic, 23(1):35-66, 1994.

[22] John F. Horty. Agency and Deontic Logic. Oxford University Press, 2001.

[23] John F. Horty. Reasoning with moral conflicts. Noûs, 37:557-605, 2003.

[24] John F. Horty and Nuel Belnap. The deliberative stit: a study of action, omission, ability, and obligation. Journal of Philosophical Logic, 24:583-644, 1995.

[25] Anthony Kenny. Will, Freedom and Power. Basil Blackwell, Oxford, 1975.

[26] Barteld Kooi and Allard Tamminga. Moral conflicts between groups of agents. Journal of Philosophical Logic, 37:1-21, 2008.

[27] E.J. Lemmon. Moral dilemmas. The Philosophical Review, 70:139-158, 1962.

[28] Ruth Barcan Marcus. Moral dilemmas and consistency. Journal of Philosophy, 77:121-136, 1980. Reprinted in [17].

[29] Joke Meheus, Mathieu Beirlaen, and Frederik Van De Putte. Avoiding deontic explosion by contextually restricting aggregation. In Guido Governatori and Giovanni Sartor, editors, DEON (10th International Conference on Deontic Logic in Computer Science), volume 6181 of Lecture Notes in Artificial Intelligence, pages 148-165. Springer, 2010.

[30] Joke Meheus, Mathieu Beirlaen, Frederik Van De Putte, and Christian Straßer. Non-adjunctive deontic logics that validate aggregation as much as possible. Under review. Preprint available at http://logica.ugent.be/centrum/writings/.

[31] J.-J. C. Meyer. A different approach to deontic logic: deontic logic viewed as a variant of dynamic logic. Notre Dame Journal of Formal Logic, 29:109-136, 1988.

[32] Graham Priest. The logic of paradox. Journal of Philosophical Logic, 8:219-241, 1979.

[33] Graham Priest. Paraconsistent logic. In Dov Gabbay and Franz Guenthner, editors, Handbook of Philosophical Logic (2nd edition), volume 8, pages 287-393. Kluwer Academic Publishers, 2002.

[34] Graham Priest. In Contradiction: A Study of the Transconsistent (2nd Edition). Oxford University Press, 2006.

[35] Geoffrey Sayre-McCord. Essays on Moral Realism. Cornell University Press, New York, 1988.

[36] Krister Segerberg. Getting started: beginnings in the logic of action. Studia Logica, 51:347-378, 1992.

[37] Yoav Shoham. A semantical approach to nonmonotonic logics. In Matthew L. Ginsberg, editor, Readings in Nonmonotonic Reasoning, pages 227-250. Morgan Kaufmann Publishers, 1987.

[38] Walter Sinnott-Armstrong. Moral Dilemmas. Basil Blackwell, Oxford/New York, 1988.

[39] Christian Straßer. An adaptive logic framework for conditional obligations and deontic dilemmas. Logic and Logical Philosophy, 19(1-2):95-128, 2010. 
[40] Christian Straßer. A deontic logic framework allowing for factual detachment. Journal of Applied Logic, 9(1):61-80, 2011.

[41] Christian Straßer, Mathieu Beirlaen, and Joke Meheus. Tolerating deontic conflicts by adaptively restricting inheritance. Logique $\mathcal{E}$ Analyse. In print. Preprint available at http://logica.ugent.be/centrum/writings/.

[42] Georg Henrik von Wright. Norm and Action. A Logical Enquiry. Routledge and Kegan Paul, London, 1963.

[43] Bernard Williams. Ethical consistency. Proceedings of the Aristotelian Society (Supplementary Volumes), 39:103-124, 1965. Reprinted in [17, 35, 44].

[44] Bernard Williams. Problems of the Self: Philosophical Papers 1956-1972. Cambridge University Press, 1973. 